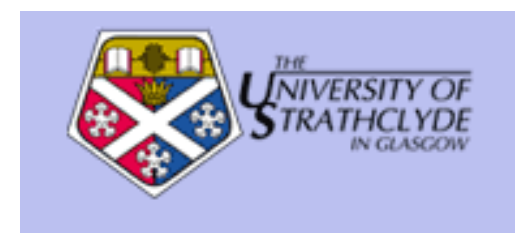

Srinil, N. and Rega, G. (2008) Space-time numerical simulation and validation of analytical predictions for nonlinear forced dynamics of suspended cables. Journal of Sound and Vibration, 315 (3). pp. 394-413. ISSN 0022-460X

http://strathprints.strath.ac.uk/18550/

This is an author produced version of a paper published in Journal of Sound and Vibration, 315 (3). pp. 394-413. ISSN 0022-460X. This version has been peer-reviewed but does not include the final publisher proof corrections, published layout or pagination.

Strathprints is designed to allow users to access the research output of the University of Strathclyde. Copyright (C) and Moral Rights for the papers on this site are retained by the individual authors and/or other copyright owners. You may not engage in further distribution of the material for any profitmaking activities or any commercial gain. You may freely distribute both the url (http://strathprints.strath.ac.uk) and the content of this paper for research or study, educational, or not-for-profit purposes without prior permission or charge. You may freely distribute the url (http://strathprints.strath.ac.uk) of the Strathprints website.

Any correspondence concerning this service should be sent to The Strathprints Administrator: eprints@cis.strath.ac.uk 


\title{
Space-Time Numerical Simulation and Validation of Analytical Predictions for Nonlinear Forced Dynamics of Suspended Cables
}

\author{
Narakorn Srinil, Giuseppe Rega \\ Department of Structural and Geotechnical Engineering, SAPIENZA University of Rome, \\ via A.Gramsci 53, Rome 00197, Italy
}

\begin{abstract}
This paper presents direct numerical simulation and validation of analytical prediction of the finite-amplitude forced dynamics of suspended cables. The main goal is to complement analytical and numerical solutions, accomplishing overall quantitative/qualitative comparisons of nonlinear response characteristics. By counting on an approximate, kinematically non-condensed, planar modeling, a simply-supported horizontal cable subject to a primary external resonance and a 1:1 (or 1:1 vs. 2:1) internal resonance is analyzed. To obtain analytical solutions, a secondorder multiple scales approach is applied to a complete eigenfunction-based series of nonlinear ordinary-differential equations of damped forced cable motion. Accounting for weakly quadratic/cubic geometric nonlinearities and multiple modal contributions, local scenarios of cable uncoupled/coupled responses and associated stability are predicted, based on chosen reduced-order models. As a cross-checking tool, direct numerical simulations of associated nonlinear partial-differential equations describing the high-dimensional, multi-degree-offreedom, system dynamics are carried out using a finite difference technique employing a hybrid explicit-implicit integration scheme. Based on system control parameters and initial conditions, cable space-time varying nonlinear responses of amplitudes, displacements and tensions are numerically assessed, thoroughly validating the analytically predicted solutions as regards actual existence, meaningful role and predominating internal resonance of coexisting/competing dynamics. Some methodological aspects are noticed, along with an insightful discussion on kinematically approximate/exact and planar/non-planar cable modeling.
\end{abstract}

Keywords suspended cable, direct numerical simulation, analytical prediction, reduced-order model, internal resonance, nonlinear forced vibration 


\section{INTRODUCTION}

Numerous research contributions have witnessed a diverse interest in geometrically nonlinear dynamics of suspended cables, with several attempts to build a reliable theoretical framework for investigating such distributed-parameter systems with quadratic and cubic nonlinearities [1]. As closed-form exact solutions capturing the actual nonlinear dynamics cannot be sought for, most of the analytical investigations have been accomplished based on some a priori hypotheses concerned with the elasto-geometrical and kinematic modeling, the mechanical equations of motion governing structural vibrations, the spatial or temporal dependence of dynamical solutions, and the initial phase-space conditions leading to particular attractors. For qualifying the richness and variability of cable nonlinear dynamic characteristics under different external and/or internal (auto-parametric) resonances, the perturbation-based multiple scales (MS) approach has largely been developed and applied to a crudely- or properly-reduced set of ordinary-differential equations (ODEs) of motion [2-9] or to the original system of partialdifferential equations (PDEs) [2-6].

To avoid some or nearly all of the aforesaid hypotheses, direct computational treatments of the approximate $[7,8]$ or exact $[10,11]$ PDEs of cable motion have recently been accomplished based on a space-time finite difference (FD) procedure confronting the finite-amplitude free vibration problems of sagged and arbitrarily inclined cables with/without internal resonances. In the meantime, several FD-based implementations have been used successfully to deal with a range of problems in nonlinear forced vibrations, including cables subject to random excitation [12], highly-extensible cable mechanics [13], low-tension cables with large displacements [14] or semi-active vibration control strategies [15]. Overall, the robustness, utility and versatility of FD algorithms have been evidenced.

However, as far as nonlinear dynamics of infinite-dimensional systems are concerned, little attention has been paid to direct numerical simulation of PDEs validating the analytical prediction of ODEs. Yet, this is a crucial aspect from both a theoretical and practical point of 
view because, when the system involves a large set of parameters due, e.g., to an internal resonance condition, the analytical approaches often fail to capture features of actual nonlinear dynamics, owing to the low-dimensional framework and several constraining assumptions. On the other hand, in addition to the prohibitive calculation costs, the accuracy of direct numerical simulation may be occasionally questionable for higher-dimensional systems, particularly in the applications where space-time varying behaviors are not easily traced out. Thus, both analytical and direct numerical solutions are of mutual significance, and relying upon solely one of them may entail incomplete or unreliable knowledge of system response.

Abhyankar et al. [16] analyzed simply-supported beams subject to a sinusoidal loading and showed a favorable comparison of chaotic responses between numerical FD (PDEs) and analytical (ODEs) solutions. Essebier and Baker [17] used spatial FDs and Runge-Kutta time integration of the ensuing ODEs to obtain undamped forced/unforced flexural responses against known analytical solutions of cantilever beams. For Euler-Bernoulli beams resting on a nonlinear elastic foundation and subject to primary/sub-harmonic resonances, Abe [18] showed that the shooting analysis of ODEs is superior to the MS analysis of associated PDEs, in comparison with FD analysis of PDEs. As far as cable nonlinear resonant oscillations are concerned, Gattulli et al. [19] used analytical and finite element discretized models of ODEs to show some superior ability of the latter in capturing higher modal contributions. In turn, based on PDEs governing undamped unforced planar vibrations, Srinil and Rega [8] have numerically checked the validity of some analytical reduced-order models for various horizontal/inclined sagged cables.

This paper aims at systematically comparing direct numerical simulations and analytical predictions of nonlinear forced dynamics of suspended cables. The main goal is to complement analytical/numerical solutions, achieving overall quantitative and qualitative comparisons of the associated response characteristics. In this framework, to reduce the analytical/computational effort, reference is made to the simpler 2-D cable model, well knowing how it can be questionable to the aim - herein not pursued - of adequately describing the overall 3-D response 
scenario. The paper is organized as follows. In Sect.2, approximate nonlinear PDEs vs. ODEs of planar motion of a simply-supported suspended cable subject to primary external and 1:1 or $2: 1$ internal resonance are summarized. Analytical and numerical solutions of ODEs and PDEs based on MS and FD methods, respectively, are presented. By focusing on horizontal cables at socalled crossovers in the natural frequency spectrum [20], Section 3 shows the analytical predictions provided by properly reduced-order models $[8,9]$, by means of frequency-response curves. The influence of several control parameters is illustrated. Depending on response amplitudes, spatial nonlinear uncoupled/coupled dynamic configurations are analytically constructed and further utilized as displacement initiations in Sect.4, where direct FD simulations of PDEs are performed, determining steady-state, multi-degree-of-freedom, responses. Various cases of $1: 1$, or $1: 1$ vs. $2: 1$, resonant modal interactions are numerically investigated to validate the analytical methodology and outcomes, as well as the pros and cons of approximate cable planar modeling. Section 5 summarizes the analyses and concludes the paper.

\section{CABLE MODEL AND SOLUTION METHODS}

Let us consider nonlinear planar damped forced vibrations of a simply-supported horizontal cable subject to a uniformly-distributed vertical harmonic excitation (Fig.1a). It is assumed that such continuous cable is linear elastic, and has moderate (low) dynamic (static) extensibility, negligible torsional, bending and shear rigidities. In a Cartesian $X-Y$ coordinate frame, the smallsagged static equilibrium $y(x)$ under gravity $g$ force is suitably described by a parabola [20] around which the cable oscillates with synchronous longitudinal $u(x, t)$ and vertical $v(x, t)$ displacements, $x(t)$ being the spatial (temporal) independent variable. In the following, the space-related (time-related) variables are non-dimensionalized with respect to cable span $X_{H}$ ( $X_{H} \sqrt{w_{C} / g H}$ ), with $H$ being the horizontal component of cable static tension and $w_{C}$ the selfweight per unit unstretched length. A prime (dot) represents partial differentiation with respect to non-dimensional position (time). 


\subsection{Nonlinear Partial/Ordinary Differential Equations of Motion}

With homogeneous boundary conditions, $u(0, t)=u(1, t)=v(0, t)=v(1, t)=0$, approximate PDEs governing finite-amplitude, damped forced, planar motion about equilibrium of suspended cables, in non-dimensional form, read [9]

$$
\begin{aligned}
& \rho \ddot{u}+c \rho \dot{u}=\left\{u^{\prime}+\frac{\alpha}{\rho^{3}}\left(u^{\prime}+y^{\prime} v^{\prime}\right)+\frac{\alpha}{\rho^{3}}\left(u^{\prime 2}+y^{\prime} u^{\prime} v^{\prime}+\frac{1}{2}\left(u^{\prime 2}+v^{\prime 2}\right)\right)+\frac{\alpha}{2 \rho^{3}}\left(u^{\prime 3}+u^{\prime} v^{\prime 2}\right)\right\}^{\prime}, \\
& \rho \ddot{v}+c \rho \dot{v}=\left\{v^{\prime}+\frac{\alpha}{\rho^{3}}\left(y^{\prime} u^{\prime}+y^{\prime 2} v^{\prime}\right)+\frac{\alpha}{\rho^{3}}\left(u^{\prime} v^{\prime}+y^{\prime} v^{\prime 2}+\frac{y^{\prime}}{2}\left(u^{\prime 2}+v^{\prime 2}\right)\right)+\frac{\alpha}{2 \rho^{3}}\left(u^{\prime 2} v^{\prime}+v^{\prime 3}\right)\right\}^{\prime}+F \rho \cos \Omega t,
\end{aligned}
$$

where $\rho=\left(1+y^{\prime 2}\right)^{1 / 2}, \alpha=E A / H$, with $E A$ being the cable axial stiffness, $c$ the viscous damping coefficient, and $F(\Omega)$ the variable amplitude (frequency) of harmonic excitation.

Equations (1-2) couple both $u$ and $v$ dynamics with parabolic equilibrium, i.e., $y=4 d x(1-x)$, in which $d$ is a cable sag-to-span ratio [20], capture geometrically quadratic/cubic nonlinearities due to cable initial curvature and axial deformation, and are valid for both (zero sag) strings and arbitrarily inclined (asymmetric) cables [21]. It is necessary emphasizing that, in contrast with the unique integro-partial differential equation of $v$ motion typically considered in cable literature based on the quasi-static stretching assumption [1-6], this kinematically noncondensed system explicitly accounts for longitudinal inertia and space-time varying dynamic tension [9]. To be generic, we keep herein exact $\rho$ values throughout Eqs.(1-2) in the subsequent analyses, whereas $\rho \approx 1$ in the associated linear terms in [9].

Casting Eqs.(1-2) in state-space form and using the orthonormality properties of linear eigenfunctions, the derived equations are then projected onto a full eigen-spectrum by letting $U^{J}=\sum_{m=1}^{\infty} f_{m} \zeta_{m}^{J}, V^{J}=\sum_{m=1}^{\infty} p_{m} \zeta_{m}^{J}, J=1-2, U^{1}=u, U^{2}=v, V^{1}=\dot{u}, V^{2}=\dot{v}, \zeta_{m}^{1}=\phi_{m}, \zeta_{m}^{2}=\varphi_{m}$, with $f_{m}\left(p_{m}\right)$ being the unknown displacement (velocity) coordinates of both $u\left(\phi_{m}\right)$ and $v\left(\varphi_{m}\right)$ shape functions of the $m$ mode of frequency $\omega_{m}$, obtained via a sine-based series [7]. The Galerkin projection is applied, thereby giving rise to a complete infinite-dimensional set of ODEs, 


$$
\dot{f}_{m}-p_{m}=0, \quad \dot{p}_{m}+2 \mu_{m} p_{m}+\omega_{m}^{2} f_{m}=\sum_{i=1}^{\infty} \sum_{j=1}^{\infty} \Lambda_{m i j} f_{i} f_{j}+\sum_{i=1}^{\infty} \sum_{j=1}^{\infty} \sum_{k=1}^{\infty} \Gamma_{m i j k} f_{i} f_{j} f_{k}+Z_{m} \cos \Omega t,
$$

for $m=1,2, \ldots, \infty$, wherein $2 \mu_{m}=c \int_{0}^{1} \rho\left(\phi_{m}^{2}+\varphi_{m}^{2}\right) d x$ and $Z_{m}=F \int_{0}^{1} \rho \varphi_{m} d x$ are modal damping and forcing terms. Expressions of the quadratic $(\Lambda)$ and cubic $(\Gamma)$ nonlinear coefficients, accounting for overall $u$ and $v$ modal shape contributions, can be found in [9]. In the following, we summarize the analytical MS solution for the nonlinear temporal behavior of ODEs (3), along with the numerical FD solution directly attacking the PDEs (1-2).

\subsection{Multiple Scales Solution with 1:1 or 2:1 Internal Resonance}

We pay our attention to the enhancing coupling effect due to planar internal resonances at meaningful crossovers [20]. To also highlight the influence of cable sag - as well as different features of mixed modal interactions - both first- and second-crossover horizontal cables are considered, the former exhibiting 1:1 resonance of first symmetric/anti-symmetric modes, whereas the latter exhibit 1:1 (2:1) resonance of second symmetric/anti-symmetric (second symmetric/first anti-symmetric) modes. To determine weakly nonlinear periodic responses and associated local stability, Eq.(3) is analyzed based on a second-order multiple scales (MS) approach capturing the slow variation of amplitudes and phases of uncoupled/coupled responses due to (higher-order) quadratic and cubic nonlinearities, damping and external/internal resonance effects [9]. With $\varepsilon$ denoting a small bookkeeping parameter (which is finally taken as 1), the damping $\mu_{m}$ and excitation $Z_{m}$ amplitudes are ordered such that they appear at the same $\varepsilon^{3}$ order, i.e., $\mu_{m} \rightarrow \varepsilon^{2} \mu_{m}$ and $Z_{m} \rightarrow \varepsilon^{3} Z_{m}$, in all resonance cases. In particular, the symmetric $(s)$ mode is the directly excited mode with $Z_{s} \neq 0$, whereas the corresponding anti-symmetric $(r)$ mode is the internally-resonant driven mode. The relationships of primary external and 1:1 internal resonances are quantified through $\Omega=\omega_{s}+\varepsilon^{2} \sigma_{f}, \omega_{s}=\omega_{r}+\varepsilon^{2} \sigma$, whereas those of primary external and 2:1 internal resonances read $\Omega=\omega_{s}+\varepsilon \sigma_{f}, \omega_{s}=2 \omega_{r}+\varepsilon \sigma$, with $\sigma_{f}$ and $\sigma$ being external and internal detuning parameters, respectively. 
The general approximate closed-form second-order solution of coupled forced damped dynamic configurations associated with both the $u(J=1)$ and $v(J=2)$ components for a 1:1 internally resonant cable is expressed as [9]

$$
\begin{aligned}
U^{J}(x, t) & \approx a_{r} \cos \left(\Omega t-\gamma_{r}\right) \zeta_{r}^{J}(x)+a_{s} \cos \left(\Omega t-\gamma_{s}\right) \zeta_{s}^{J}(x)+ \\
& \frac{1}{2}\left\{\begin{array}{l}
a_{s}^{2}\left[\cos \left(2 \Omega t-2 \gamma_{s}\right) \psi_{s s}^{J}(x)+\kappa_{s s}^{J}(x)\right]+a_{r}^{2}\left[\cos \left(2 \Omega t-2 \gamma_{r}\right) \psi_{r r}^{J}(x)+\kappa_{r r}^{J}(x)\right]+ \\
a_{s} a_{r}\left[\cos \left(2 \Omega t-\gamma_{r}-\gamma_{s}\right) \psi_{r s}^{J}(x)+\cos \left(\gamma_{r}-\gamma_{s}\right) \kappa_{r s}^{J}(x)\right]
\end{array}\right\},
\end{aligned}
$$

whereas that for a 2:1 internally resonant cable reads [9]

$$
\begin{aligned}
& U^{J}(x, t) \approx a_{r} \cos \left(\frac{\Omega t}{2}-\frac{\gamma_{s}}{2}-\frac{\gamma_{r}}{2}\right) \zeta_{r}^{J}(x)+a_{s} \cos \left(\Omega t-\gamma_{s}\right) \zeta_{s}^{J}(x)+ \\
& \frac{1}{2}\left\{\begin{array}{l}
\left.a_{s}^{2}\left[\cos \left(2 \Omega t-2 \gamma_{s}\right) \psi_{s s}^{J}(x)+\kappa_{s s}^{J}(x)\right]+a_{r}^{2}\left[\cos \left(\Omega t-\gamma_{s}-\gamma_{r}\right) \psi_{r r}^{J}(x)+\kappa_{r r}^{J}(x)\right]+\right] \\
a_{s} a_{r}\left[\cos \left(\frac{3}{2} \Omega t-\frac{3}{2} \gamma_{s}-\frac{1}{2} \gamma_{r}\right) \psi_{r s}^{J}(x)+\cos \left(\frac{1}{2} \Omega t-\frac{1}{2} \gamma_{s}+\frac{1}{2} \gamma_{r}\right) \kappa_{r s}^{J}(x)\right]
\end{array}\right\} .
\end{aligned}
$$

Here, $\gamma_{r}=\left(\sigma_{f}+\sigma\right) t-\beta_{r}, \gamma_{s}=\sigma_{f} t-\beta_{s}$ in Eq.(4), whereas $\gamma_{r}=\sigma t-2 \beta_{r}+\beta_{s}, \gamma_{s}=\sigma_{f} t-\beta_{s}$ in Eq.(5), with $\beta_{r}\left(\beta_{s}\right)$ being the phase of associated $a_{r}\left(a_{s}\right)$ amplitude. In addition to the first-order superimposition of resonant $\left(\zeta_{r}^{J}, \zeta_{s}^{J}\right)$ modal functions with their correlated phases (e.g., Figs.1b, c), the spatial displacement distributions in both Eqs.(4) and (5) further depend on second-order shape functions assembling quadratic nonlinear effects of every retained resonant/non-resonant mode via $\psi_{i j}^{J}, \kappa_{i j}^{J}$ [6]. Similarly, second-order uncoupled dynamic configurations due to the solely primary resonance of symmetric mode can be extracted from Eq.(4) or (5). Meaningful temporal dependence and variation of second-order uncoupled $\left(a_{s}\right)$ and coupled $\left(a_{r}-a_{s}\right)$ amplitudes plus their relative phases $\left(\gamma_{r}, \gamma_{s}\right)$ are enforced through the ensuing four-dimensional modulation equations [9], viz., for a 1:1 resonant modal interaction,

$$
\begin{aligned}
& \dot{a}_{r}=-\mu_{r} a_{r}+\frac{K a_{s}^{2} a_{r} \sin \left(2 \gamma_{r}-2 \gamma_{s}\right)}{8 \omega_{r}}, \\
& a_{r} \dot{\gamma}_{r}=\left(\sigma_{f}+\sigma\right) a_{r}+\frac{K_{r r} a_{r}^{3}}{8 \omega_{r}}+\frac{K_{r s} a_{r} a_{s}^{2}}{8 \omega_{r}}+\frac{K a_{s}^{2} a_{r} \cos \left(2 \gamma_{r}-2 \gamma_{s}\right)}{8 \omega_{r}} \\
& \dot{a}_{s}=-\mu_{s} a_{s}-\frac{K a_{r}^{2} a_{s} \sin \left(2 \gamma_{r}-2 \gamma_{s}\right)}{8 \omega_{s}}+\frac{Z_{s} \sin \gamma_{s}}{2 \omega_{s}}
\end{aligned}
$$




$$
a_{s} \dot{\gamma}_{s}=\sigma_{f} a_{s}+\frac{K_{s s} a_{s}^{3}}{8 \omega_{s}}+\frac{K_{r s} a_{s} a_{r}^{2}}{8 \omega_{s}}+\frac{K a_{r}^{2} a_{s} \cos \left(2 \gamma_{r}-2 \gamma_{s}\right)}{8 \omega_{s}}+\frac{Z_{s} \cos \gamma_{s}}{2 \omega_{s}},
$$

whereas for a 2:1 resonant modal interaction,

$$
\begin{aligned}
& \dot{a}_{r}=-\mu_{r} a_{r}+\frac{\mathfrak{R} a_{r} a_{s} \sin \gamma_{r}}{4 \omega_{r}}, \\
& a_{r}\left(\dot{\gamma}_{r}+\dot{\gamma}_{s}\right)=\left(\sigma_{f}+\sigma\right) a_{r}+\frac{\mathfrak{R} a_{r} a_{s} \cos \gamma_{r}}{2 \omega_{r}}+\frac{K_{r r} a_{r}^{3}}{4 \omega_{r}}+\frac{K_{r s} a_{r} a_{s}^{2}}{4 \omega_{r}}, \\
& \dot{a}_{s}=-\mu_{s} a_{s}-\frac{\mathfrak{R} a_{r}^{2} \sin \gamma_{r}}{8 \omega_{s}}+\frac{Z_{s} \sin \gamma_{s}}{2 \omega_{s}} \\
& a_{s} \dot{\gamma}_{s}=\sigma_{f} a_{s}+\frac{\mathfrak{R} a_{r}^{2} \cos \gamma_{r}}{8 \omega_{s}}+\frac{K_{s s} a_{s}^{3}}{8 \omega_{s}}+\frac{K_{r s} a_{s} a_{r}^{2}}{8 \omega_{s}}+\frac{Z_{s} \cos \gamma_{s}}{2 \omega_{s}} .
\end{aligned}
$$

As discussed in [6-9], the non-trivial $K$ or $\mathfrak{R}$ entail relevant 1:1 or 2:1 resonance activation because of vanishing nonlinear orthogonality properties of resonant modes. Depending on control parameters, $\left(\sigma, \sigma_{f}, \mu, F\right)$, both (6)-(9) and (10)-(13) admit both uncoupled and coupled fixed-point $\left(\dot{a}_{r}=\dot{a}_{s}=\dot{\gamma}_{r}=\dot{\gamma}_{s}=0\right)$ solutions [9]. Overall interaction coefficients $\left(K, \mathfrak{R}, K_{r r}, K_{s s}\right.$, $\left.K_{r s}\right)$ - accounting for quadratic/cubic nonlinearities and infinite-dimensional modal contributions - can be found in [9] with comprehensive convergence analyses establishing properly reducedorder models.

\subsection{Space-Time Finite Difference Solution}

Direct numerical simulations of non-dimensional PDEs (1-2) governing an initial-boundary value problem of cable damped forced resonant motion are carried out by employing a secondorder finite-difference (FD) approach centrally approximating both spatial and temporal derivatives, thanks to a relevant straightforward routine developed by the authors in $[10,11]$ to handle cable large-amplitude 3-D free vibrations. For the considered 2-D vibration problems, the continuous cable is divided into $N$ equal space segments, which entails solving simultaneously a 2(N-1) multi-degree-of-freedom system for nonlinearly coupled $u$ and $v$ nodal vectors. Partitioning the time into a series of incremental steps, a hybrid explicit-implicit numerical 
integration scheme is adopted via a predictor-corrector iterative implementation and a specified tolerance controlling global solution stability and convergence at each time-step.

As far as initial state-space conditions are concerned, we assign zero velocities but different spatial displacement options. In so doing, we utilize the known MS-based spatial distributions of uncoupled/coupled displacements (Eq.4 or 5). Apart from establishing a link between numerical and analytical solutions, this allows us to determine (i) how nonlinear spatial MS solutions actually evolve with time and (ii) how much computational effort is needed in reaching a steadystate time response of a high-dimensional discretized system, with respect to the conventional zero initiation (i.e., cable at rest). Considering the same control parameters, it further allows us (iii) to validate the MS prediction against FD space-time laws with regard to the chosen reducedorder model embedded in the amplitude and displacement solutions (Eqs.4-13) [9], to the actual existence and role of stable/unstable, uncoupled/coupled, 1:1/2:1 resonant, equilibrium/periodic (constant/varying-amplitude) motion, and to the validity of asymptotic MS solutions when considering greater response amplitudes. Moreover, (iv) insightful multi-degree-of-freedom spatial comparisons of MS vs. FD maximum dynamic displacements are of practical interest for accurate dynamic tension estimations, by also accounting for the approximate vs. exact [11] kinematically non-condensed modeling.

\section{ANALYTICAL PREDICTION VIA CHOSEN REDUCED-ORDER MODELS}

By considering $X_{H}=850 \mathrm{~m}, E A \approx 20,792,460 \mathrm{kN}, w_{C} \approx 9.48 \mathrm{kN} / \mathrm{m}$ as in [7-9], the elastogeometric $(\alpha=E A / H, d)$ dimensionless parameters of first- and second-crossover cables are $(\alpha=$ 642.72, $d=0.031)$ and $(\alpha=1024.28, d=0.050)$, respectively. The first-crossover cable exhibits (nearly-tuned) $1: 1$ internal resonance with $\omega_{s=2} \approx 6.287$ and $\omega_{r=1} \approx 6.252$, whereas the secondcrossover cable exhibits either (nearly tuned) $1: 1$ or $2: 1$ internal resonance with $\omega_{s=4} \approx 12.503$ and $\omega_{r=3} \approx 12.498$ or $\omega_{s=4}$ and $\omega_{r=1} \approx 6.205$, with the relevant linear orthonormalized $(r, s)$ shape functions being displayed in Figs.1b and c, respectively. As regards reduced-order models of 
amplitude/displacement solutions, a series of modal contribution and convergence analyses of second-order quadratic coefficients has been conducted as in [8, 9]. As already discussed in [9], for crossover cables one may omit a priori negligible contributions of anti-symmetric nonresonant modes, whereas meaningful contributions of symmetric non-resonant modes have to be accounted for along with those of the two resonant modes. Accordingly, with $M$ being the highest order of retained modes through the overall nonlinear coefficients in Eqs.(4-13), we have chosen $M=10$ (without non-resonant anti-symmetric modes) for first-crossover cable and $M=$ 15 (with all modes, to possibly account for also a multiple internal resonance [9]) for secondcrossover cable.

\subsection{Frequency-Response Diagrams and Influence of Control Parameters}

Based on the Cartesian version of modulation Eqs.(6-9) or (10-13), a series of frequencyresponse diagrams, whose bifurcations discriminate coupled from uncoupled (as well as fixed point from limit cycle) solution, is parametrically obtained via a continuation approach [22], which has been verified by corresponding Runge-Kutta integration solutions [9, 21]. For the sake of ease in FD simulations of all resonance cases (Sect.4), we assume the modal damping $\mu=\mu_{r}$ $=\mu_{s}$ in such a way that the damping coefficient $c$ can be evaluated as a single-valued parameter to be given in the associated PDEs (1-2). In the following, solid lines denote stable fixed points, whereas dashed (dotted) lines denote unstable fixed points settled down through saddle-node SN or pitchfork PF (Hopf) bifurcations.

The first-crossover cable involving 1:1 internal resonance is first analyzed. Three different cases - which assume perfect tuning of resonant frequencies, i.e., $\sigma=0$ - are considered, viz., (i) $\mu=.005, F=.005$, (ii) $\mu=.05, F=.005$, (iii) $\mu=.005, F=.010$, in order to examine the effect of varying damping (ii) or forcing (iii) parameter with respect to the reference (i) case. Associated frequency-response curves of coupled $a_{r}$ (internally driven) and $a_{s}$ (externally excited), and uncoupled $a_{s}$ amplitudes are plotted in Figs.2a, b and c, respectively. To ascertain 
the possible $\sigma$ effect, continuation of the (ii) case is also made with actual - albeit small - value of $\sigma\left(\omega_{s}-\omega_{r} \approx .005\right)$, and only the coupled $a_{r}-a_{s}$ results are traced out in Figs.2a and b. Depending on relative contributions of quadratic and cubic nonlinearities, overall frequency sweeping analyses in Fig.2 highlight how unstable coupled $a_{s}$ branches (2b) emanate from uncoupled $a_{s}$ ones (2c) via double $\mathrm{PF}_{1}$ and $\mathrm{PF}_{2}$ bifurcations. Hysteresis (jump) phenomena are found in all uncoupled (2c) and coupled (2a and b) branches with multiple SN bifurcations, and the leaningbackbone softening behavior due to predominant quadratic nonlinearities is manifested in uncoupled branches $(2 \mathrm{c})$.

Due to activation of 1:1 internal resonance, stable (as well as unstable) coupled $a_{r}$ and $a_{s}$ amplitudes occur over a wide $\sigma_{f}$ range (Figs.2a and b), with $a_{r}\left(a_{s}\right)$ prevailing right (left) of the case of perfect primary resonance $\left(\sigma_{f}=0\right)$. It should also be noted that right (left) of the $\mathrm{PF}_{2}$ $\left(\mathrm{PF}_{1}\right)$ bifurcation, the uncoupled (coupled) $a_{s}$ amplitudes are greater than the corresponding coupled (uncoupled) $a_{s}$ ones [9], and there is a range, bounded by $\mathrm{PF}_{1}$ and $\mathrm{PF}_{2}$ bifurcations, where only stable coupled $a_{r}-a_{s}$ solutions exist, the larger (smaller) the $F(\mu)$ the greater the ensuing region. For weaker-damped cases (i and iii), a marginal range (A or B) between Hopf bifurcations to which limit cycles are amenable is observed, being enlarged with increasing $F$. By increasing the damping by an order of magnitude $(\mu=.05)$, i.e., case (ii) vs. (i), both amplitude responses and modal interaction effect are reduced due to a greater energy dissipation. In turn, considering the actual $\sigma=.005$ value quantitatively - though slightly - influences the driven $a_{r}$, rather than the excited $a_{s}$, with respect to the associated case (ii) with $\sigma=0$. Yet, overall qualitative features practically remain the same in both $\sigma$ cases, with a slight shift of SN and PF bifurcations. Accordingly, it appears sufficient to consider $\sigma=0$ in the analytical solution.

Modal interactions in second-crossover cable are now discussed in Fig.3. By assigning the same parameters $\sigma=0, \mu=.005, \mathrm{~F}=.005$ as in case (i) of first-crossover cable (Fig.2), coupled $a_{r}$ and $a_{s}$ responses due to $1: 1$ or $2: 1$ resonance are independently traced out but are displayed 
altogether in Figs.3a and b, respectively. The trend of both response curves qualitatively resembles that in Fig.2: the coupled branches originate from associated uncoupled ones (not shown), with PF, SN and Hopf (region C or D) bifurcations occurring at certain $\sigma_{f}$ parameters. However, here, both 1:1 and 2:1 resonant interactions persist throughout the considered $\sigma_{f}$ range. The predominant role is played by either the driven $a_{r}$ or the excited $a_{s}$ amplitude in $1: 1$ resonant responses, depending on the sweeping $\sigma_{f}$ parameter as in Fig.2, whereas the driven $a_{r}$ substantially prevails over the excited $a_{s}$ in 2:1 resonant responses, regardless of $\sigma_{f}$. This means that the $a_{r}$ mode behaves as an absorber with respect to the $a_{s}$ mode, the energy being transferred from the latter to the former owing to $2: 1$ resonance. Such circumstance will be justified via numerical analyses in Sect 4, along with the actual predominant role played by $2: 1$ vs. 1:1 resonant interactions and the validity of $\sigma=0$ assumption.

\subsection{Mixed/Symmetric Nonlinear Dynamic Displacements}

By focusing on stable uncoupled/coupled amplitude solutions (Fig.2 or 3), corresponding nonlinear dynamic $u-v$ displacements can be analytically constructed through Eq.(4) or (5). As direct FD simulations and spatial MS-FD displacement comparisons in Sect.4 involve a multipledimensional phase space spanned with $u$ and $v$ amplitudes (as well as their velocities) of every cable nodal vector, it is worth examining the spatio-temporal varying displacements of the cable when one of its nodal - essentially $v$-components, contributing meaningfully to both uncoupled and coupled displacements, reaches its maximal amplitude either in the downward $\left(\mathrm{Y}^{+}\right)$or upward $\left(\mathrm{Y}^{-}\right)$direction (Fig. 1a). The cable mid-span $(x=0.5)$ or quarter-span from left support ( $x$ $=0.26)$ is preferably assumed as such a benchmark point in first- and second-crossover cable analyses, respectively.

Considering first-crossover cable with $\mu=.05, F=.005$ (Fig.2c), uncoupled $v$ displacements $\left(\mathrm{Y}^{+}, \mathrm{Y}^{-}\right)$corresponding to $\sigma_{f}\left(a_{s}\right)=-0.3(.001094)$ and $0.1(.002249)$ are comparatively shown in Fig.4a. The spatial resemblance to the primary-resonant first symmetric mode (Fig.1b) is 
apparent, and the upward-downward (e.g., mid-span) amplitude differences (drifts) due to second-order spatial corrections of all retained (higher-order) symmetric modes [9] are observed, being enhanced with increasing $a_{s}$. As the 1:1 resonant interaction occurs (Figs.2a, b), the coupled mixed-modal $v$ displacement $\left(\mathrm{Y}^{+}, \mathrm{Y}^{-}\right)$profiles are evidently asymmetric, with two opposite unequal curvatures, as shown in Fig.4b $\left(\sigma_{f}=-0.1, F=.005\right)$ governing the cases (i) $\mu$ $=.005$ and (ii) $\mu=.05$ with $\left(a_{r}, a_{s}\right)=(.002186, .003842)$ and $(.001972, .003726)$, respectively. In comparison with Fig.4a, spatial drifts in Fig.4b are more noticeable as a result of modal interaction.

As to second-crossover cable, it is worth distinguishing the coupled $v$ configurations due to 1:1 resonance from those due to $2: 1$ resonance, by considering, for instance, $\sigma_{f}=-0.1$ in Fig.3. Because of the coupled amplitude $a_{s}(.001163)$ being greater than $a_{r}(.000634)$, the $1: 1$ resonant $\left(\mathrm{Y}^{+}, \mathrm{Y}^{-}\right)$displacements in Fig.4c look similar to the directly-excited second symmetric mode (Fig.1c), though being asymmetric due to the second anti-symmetric modal participation leading to some local curvature changes. On the other hand, the $2: 1$ resonant $\left(\mathrm{Y}^{+}, \mathrm{Y}^{-}\right)$displacements in Fig.4d are nearly - but not completely - dominated by the driven lowest anti-symmetric mode since $a_{r}(.001237)$ is considerably greater than $a_{s}(.000212)$. As long as $a_{r}$ amplitudes (Fig.3a) are the most significant contributions to the coupled responses (Fig.3), such spatial prevalence of the indirectly-excited mode remains qualitatively the same, albeit sweeping $\sigma_{f}$ towards left or right. Yet, second-order spatial corrections are observable, e.g., at quarter span, where the opposite amplitude values are unequal. In the following, the main uncoupled/coupled spatial characters of MS-based $v$ displacements in Fig. 4 will be recognized in numerical simulations, on the basis of their steady and maximum response amplitudes.

\section{DIRECT NUMERICAL SIMULATION AND VALIDATION}

Depending on control parameters, overall amplitude and displacement MS predictions in Sect. 3 are now validated by direct FD simulations. After some convergence tests, it was chosen 
to use 50 cable discrete elements with $\Delta x=0.02$ and a general time step equal to $0.0001 \mathrm{sec}$., the simulations being unconditionally stable as long as small oscillating amplitudes are considered and cables are prevented from compressive total stresses [11]. The new dimensionless time variable $T$, in which original time is non-dimensionalized with respect to the forcing period, is considered. Depending on initial $u$ and $v$ displacements (zero velocities), a series of spacevarying time histories is carried out, capturing both transient and steady (fast and slow) dynamics over repeated cycles $T$. For the sake of MS-FD comparisons, histories of spatial $\left(\mathrm{Y}^{+}, \mathrm{Y}^{-}\right)$steady FD displacements, corresponding to the aforesaid benchmark points $(x=0.5$ or 0.26$)$ at their maxima, are determined in the 1:1 or 2:1 resonance cases. In the following, reference is made to steady amplitude (Figs.2 and 3) or spatial displacement (Fig.4) MS results by assuming perfect internal resonance $\sigma=0$, as $\sigma$ produces a minor (negligible) quantitative (qualitative) effect (Sect.3.1). Yet, whenever desirable, the actual nonlinear $\sigma$ value can be evaluated a posteriori via a frequency domain analysis of the obtained FD responses (see end of Sect.4.2).

\subsection{Dependence of Uncoupled/Coupled Nonlinear Responses on Initial Conditions}

As nonlinear dynamic response depends, in general, on initial conditions, it is worth examining such dependence as regards both uncoupled and coupled responses, by also accounting for the effect of control parameters. By way of example, first-crossover cable is analyzed. Letting $\mu=.05, F=.005, \sigma_{f}=0.1$ (Fig.2c), Figs.5a and b illustrate FD simulations of mid-span $v$ amplitudes obtained with (a) zero and (b) non-zero MS-based uncoupled $\mathrm{Y}^{-}$(solid lines) or $\mathrm{Y}^{+}$(dotted lines) spatial initiations (Fig. 4a). It can be seen that, irrespective of initiations and transient features, all responses reach their steady states with a comparable running time. With $\mu=.005$, the reduced damping role is next discussed through Figs.5c-e based on zero initiations. With $F=.005$ and $\sigma_{f}=0.1$, Fig.5c exhibits a longer transient than that in Fig.5a or b, even though all cases correspond to nearly-equal $a_{s}$ MS amplitudes (Fig.2c), which entail comparable steady FD responses. Such long transients still persist with $\sigma_{f}=-0.3$ (Fig.5d), 
with the ensuing steady response exhibiting a smaller $a_{s}$ regime in agreement with MS prediction (Fig.2c), and they become even more remarkable, indeed also in terms of larger (transient/steady) amplitudes, in Fig.5e when increasing the forcing to $F=.01$. These results show the capability of FD simulation to account for $\mu$ and $F$ effects on transient outcomes.

Nonetheless, directly initiating the associated spatial (e.g., $\mathrm{Y}^{-}$) uncoupled MS configuration (Fig.5f) entails reducing significantly (approximately by half) the overall transient time with respect to that in Fig.5e. This highlights that, even though both FD simulations (5e and f) ultimately yield the same steady outputs - revealing also a slight drift due to quadratic nonlinearities (e.g., Fig.4) -, utilizing the spatial MS-based uncoupled displacements as direct initiations in FD analysis is useful for saving computational time. This is reasonable because the predicted steady MS amplitudes (Fig.2c) actually govern the slow-varying dynamics via modulation equations. Because of small $a_{s}$ amplitudes, the corresponding spatial FD distributions (not displayed) of all responses in Fig.5 show no feature of modal interaction, thus confirming the occurrence of uncoupled solutions characterized by the primary-resonant first symmetric mode, similar to that constructed in Fig.4a.

Depending on spatial initiations, it is now necessary understanding how responses actually evolve as the 1:1 resonant coupling comes into play. To this end, first-crossover cable is again considered in Fig. 6 with $\mu=.005, F=.005$ and $\sigma_{f}=-0.1$, for which MS analysis entails only stable coupled solution in Fig.2. To discriminate spatially coupled (asymmetric) from uncoupled (symmetric) $v$ responses (e.g., Figs.4b vs. a) throughout the time running, FD responses at about cable quarter-span from left $(x=0.2)$ and right $(x=0.8)$ supports are comparatively recorded with black and grey lines, respectively, along with the corresponding spatial displacements during a period of peak-to-peak steady amplitudes.

First of all, zero initiations are considered and some qualitative differences between transient and steady responses are revealed in Fig.6a. At the beginning, both quarter-span responses have equal amplitudes; thus spatial responses are associated with first-symmetric mode due to the 
solely primary resonance. However, the spatial symmetry is destroyed at $T \approx 300$ after which the anti-symmetric mode is periodically driven into the response due to actual activation of 1:1 resonance eventually giving rise to unequal-amplitude steady responses, with the right quarterspan one becoming greater than the other. Clear combination of resonant symmetric/antisymmetric displacements is evidenced, which entails different profiles with respect to the corresponding MS $\left(\mathrm{Y}^{+}, \mathrm{Y}^{-}\right)$ones in Fig. $4 \mathrm{~b}$ as regards the relative phases. This may be attributed to the fact that the MS analysis relies upon constrained modal phases ensuing from the linear eigenfunctions (Fig.1b), which affect the analytical spatial displacements, Eq.(4), whereas direct FD simulations with zero initiations are independent of such constraints.

The development of coupled from initiated uncoupled response is now discussed. Because no stable uncoupled MS solution exists at $\sigma_{f}=-0.1$ (Fig.2c), spatial uncoupled MS configurations at a neighboring frequency value $\left(\sigma_{f}=0.1\right)$, similar to Fig.4a, are utilized. By initiating with the phase-different $\mathrm{Y}^{-}$and $\mathrm{Y}^{+}$uncoupled MS configurations, remarkably quantitative as well as qualitative differences arise between Figs.6b and c, respectively. In particular, the responses in Fig.6b do not signal any modal coupling feature, maintaining the symmetric displacements, and they rapidly reach steady regimes. On the contrary, Fig.6c highlights that the initiated uncoupled responses are no longer stable after some transient periods $(T \approx 200)$, where the mixed modal interaction due to $1: 1$ resonance comes into play, affecting the spatial transition behavior from the imposed symmetric uncoupled to steady asymmetric coupled responses. These FD results reveal how the onset of coupled responses depends on the phases of initiated uncoupled MS displacements. Yet, the left (right) quarter-span steady response in Fig.6c is greater (smaller), thereby producing resonant profiles differing, again in terms of relative phases, from those in Fig.6a, although both of them evidence coupled dynamics.

Not only do the spatial phases of uncoupled initiations, but also those of coupled initiations, affect the FD outcomes. By directly initiating with the phase-different $\mathrm{Y}^{-}$vs. $\mathrm{Y}^{+}$coupled MS configurations at $\sigma_{f}=-0.1$ (i.e., Fig.4b), relevant steady FD responses entail again meaningful 
phase-different coupled dynamics in Figs.6d vs. 6e, even if both initiations rely on the same MS amplitude solution. In essence (Fig.6d), initial transient periods are substantially eliminated and corresponding coupled profiles are similar to those in Fig.6c. Conversely, longer transients are observed in Fig.6e exhibiting also the initial phase exchange that makes the ensuing coupled profiles similar to those in Fig.6a. Thus, depending on spatial initiations and associated phases, actual FD responses in Fig.6 allow us to identify, regarding steady spatial displacements, three coexisting numerical solutions consisting of either one uncoupled (6b) or two coupled (6a and e or $6 \mathrm{c}$ and d) responses, in contrast with the uniquely constrained spatial MS solution of Fig.4b. In particular, zero spatial initiation is seen to lead to the former coupled response, likely in connection with the spatial response drift towards $\mathrm{Y}^{-}$direction observed in Fig.4b. Towards the aim of comparing individually MS-FD spatial distributions, attention is turned to the case of spatial $\mathrm{Y}^{-}$coupled initiations (Fig.6d) because, besides leading to dynamic coupled profiles qualitatively resembling those predicted by MS solution (Fig.4b), it does not need a mammoth calculation task with respect to other initiation cases. This will be addressed in Sect.4.2.

Overall, the actual existence of amplitude-steady uncoupled/coupled damped forced FD responses validates the fixed-point MS predictions based on reduced-order models. Yet, the space-time varying FD analyses highlight the influence of spatial initiations and associated phases on the spatial numerical outcomes being actually governed by either the solely primary resonant or the primary/internally resonant dynamics. Meaningful transient and dynamicinstability mode-transition characteristics are revealed. Against the case of zero initiations, utilizing proper MS-based spatial configurations as FD initiations considerably reduces the computational CPU time in reaching steady-state responses. This numerical aspect appears practically advantageous when handling weakly damped multi-degree-of-freedom resonant responses. 


\subsection{Role of Coexisting Resonant Dynamics and Spatial Displacement Comparisons}

Due to coexistence of uncoupled/coupled solutions (Fig.2), 1:1 vs. 2:1 internally-resonant modal interactions (Fig.3) and possible amplitude-modulated solutions (Figs.2 and 3), it is worthwhile assessing the actual role of such analytically-predicted coexisting dynamics - when varying control parameters - via direct numerical simulations based on relevant initiations. Of practical concern, overall quantitative and qualitative comparisons of MS vs. FD solutions are thoroughly made in terms of (steady-state) spatial $\left(\mathrm{Y}^{+}, \mathrm{Y}^{-}\right) v$ displacement distributions meaningful for reliable dynamic stress estimations (see Sect.4.3).

By considering first-crossover cable with different sets of $\left(\mu, F, \sigma_{f}\right)$, Fig.7 compares 1:1 resonant configurations between MS (lines) and FD (circles) solutions. As anticipated in Sect.4.1, all FD simulations in Fig.7 are obtained with the fixed $\mathrm{Y}^{-}$spatial initiation of MS coupled solution with $\mu=.005, F=.005, \sigma_{f}=-0.1$. Relevant to Figs.6d and 4b, Fig.7a provides a good qualitative and quantitative agreement of asymmetric nonlinear $\mathrm{Y}^{+}$and $\mathrm{Y}^{-}$resonant profiles, in terms of both amplitudes and relative phases. This holds satisfactorily also in the case of increased $\mu=0.05$ (Fig.7b) or $F=0.01$ (Fig.7c), while keeping other parameters fixed. Then, $\sigma_{f}$ is varied towards increasing $a_{r}$ - $a_{s}$ amplitudes (Figs.2a and b). It is seen that all of the FD outcomes in Figs.7d $\left(\sigma_{f}=-0.2\right), 7 \mathrm{e}\left(\sigma_{f}=-0.3\right)$ and $7 \mathrm{f}\left(\sigma_{f}=-0.4\right)$ validate analytical MS predictions regarding the applicable range of asymptotic amplitudes. It is also worth noting that stable uncoupled MS responses, though coexisting in such $\sigma_{f}$ range, do not play any role since coupled MS responses (Fig.6d) are initiated in FD simulations.

The actually prevailing role played by uncoupled responses against coupled ones is now highlighted. Indeed, when being outside the predicted range in between $\mathrm{PF}_{1}$ and $\mathrm{PF}_{2}$ bifurcations in Figs.2a and b, it follows from Figs.5b and $f$ that steady FD responses are uncoupled when uncoupled MS solutions are initiated (Fig.2c). However, such a persistence of spatial shape initiation does not occur any more when initiating with coupled MS solutions, as shown in Figs.8a $(F=.005)$ and $8 \mathrm{~b}(F=.01)$ with $\mu=.005$. By increasing $\sigma_{f}$ right of $\mathrm{PF}_{2}$ bifurcation, both 
quarter-span FD $v$ responses in Figs.8a $\left(\sigma_{f}=0\right)$ and $8 \mathrm{~b}\left(\sigma_{f}=0.1\right)$ tend, after variable amplitude transients due to asymmetric coupled initiations, to comparable values associated with spatially symmetric vibration $\left(\mathrm{Y}^{+}, \mathrm{Y}^{-}\right)$profiles (circles) typical, of course, of the first-symmetric mode and resembling very much those (solid lines) predicted by MS solution. Quantitative differences are practically less than those of coupled responses (Fig.7), which experience a higher-dimensional mixed modal interaction. When being away from any modulated (periodic) solution, such circumstance occurs even if sweeping $\sigma_{f}$ further away to the right or initiating with $a_{r}$-dominant coupled MS configurations at the associated $\sigma_{f}$. This predominance of uncoupled FD responses right of $\mathrm{PF}_{2}$ bifurcation is anyhow related to MS predictions in Fig.2, whose uncoupled $a_{s}$ amplitudes (2c) are greater than the corresponding coupled ones (2b), implying that the primary resonance plays a greater role than 1:1 resonance. It is the opposite case of Fig.7 where, sweeping $\sigma_{f}$ left of $\mathrm{PF}_{2}$ bifurcation, coupled FD responses settle down when initiating coupled MS responses, as the predicted coupled (uncoupled) $a_{s}$ amplitudes are considerably greater (smaller).

Yet, by continuously sweeping $\sigma_{f}$ farther below the left SN, such steady coupled responses in Fig.7 do not occur anymore because of a jump phenomenon. As predicted in Fig.2, e.g., with a fixed $F=.005$, the coupled MS solution shows how the cable with meaningful $(\mu=.05)$ or small $(\mu=.005)$ damping loses stability via $\mathrm{SN}$ at plausibly small $\left(\sigma_{f} \approx-0.3035\right)$ or meaningless large ( $\left.\sigma_{f} \approx-30\right)$ response $a_{r}-a_{s}$ amplitudes, respectively. Accordingly, the FD simulations with coupled MS initiations validate $(\mu=.05)$ or strongly revise $(\mu=.005)$ such predictions of jump phenomena by highlighting the occurrence of steady small-amplitude uncoupled responses at $\sigma_{f}$ $\approx-0.33$ in Figs.9a $(\mu=.05)$ and $\sigma_{f} \approx-0.77$ in Fig. $9 \mathrm{~b}(\mu=.005)$.

In turn, the actual existence of nonlinearly periodic response associated with a predicted Hopf bifurcation is also ascertained. By focusing on the coupled branches in Figs.2a and b with $\mu=.005$ and $F=.01$, the MS local stability shows that two Hopf bifurcations delimit a marginal 
$\sigma_{f}$ range $\left(\sigma_{f} \approx 0.0429\right.$ and 0.0836$)$. The FD simulations in Figs.10a and b validate this prediction by highlighting, besides transient dynamics, the steadily amplitude-modulated responses governing coupled $u(10 \mathrm{a})$ and $v(10 \mathrm{~b})$ amplitudes at $\sigma_{f}=0$, with assigned spatial coupled initiations as in Figs. 7 and 8.

The second-crossover slacker cable is now analyzed, towards the aim of understanding - via FD simulations - whether $1: 1$ or $2: 1$ resonance activation actually predominates in the dynamic response as their independent MS solutions coexist in the whole considered $\sigma_{f}$ range in Fig.3, with identical $\mu=.005$ and $F=.005$. Recall also that cable node at $x=0.26$ is assumed as the benchmark point concerning spatial $\left(\mathrm{Y}^{+}, \mathrm{Y}^{-}\right)$displacements. Following Sect.4.1, a similar analysis - aimed at determining proper spatial initiations for coupled FD responses - has been performed, ending up with the choice to use, for a pertinent $\sigma_{f}$, spatial $\mathrm{Y}^{-}$coupled $1: 1$ (like firstcrossover cable) and $\mathrm{Y}^{+}$coupled 2:1 resonant MS displacements (e.g., Figs.4c and d) as initiations in the relevant FD cases. Upon varying $\sigma_{f}$, for each spatial 1:1 or 2:1 resonant initiation, the associated steady-state FD outcomes of $v$ amplitudes at $x=0.26$ are comparatively zoomed in Fig.11 with solid lines (1:1) or circles (2:1), respectively.

Starting with $\sigma_{f}=-0.4$, both FD simulations in Fig.11a show qualitatively different, though quantitatively similar, steady states, regarding the relative phases, owing to different initiations. At $\sigma_{f}=-0.2$, the two responses coincide (Fig.11b), with smaller amplitudes than those in Fig.11a, as predicted in Fig.3. To gain overall insight into global responses, the relevant $\left(\mathrm{Y}^{+}, \mathrm{Y}^{-}\right)$ displacement comparisons are depicted in Fig.12a, in which both 1:1 (solid lines) and 2:1 (circles) resonant FD simulations are plotted against MS (dashed lines) 2:1 resonant results (e.g., Fig.4d). It can be seen that, apart from a quantitative deviation of MS displacements from the other two perfectly-matched FD displacements, all of them put into evidence the nearlypredominating lowest anti-symmetric mode due to the major role of 2:1 - against 1:1 resonance, in accordance with what observed in Fig.3a. This highlights how the coexisting 1:1 
resonance does not play any role even when directly initiating with spatial 1:1 resonant displacements. Such circumstance holds even when sweeping $\sigma_{f}$ right of $\sigma_{f}=0$ in Fig.3, as shown in Figs.11c $\left(\sigma_{f}=0.2\right)$ and $11 \mathrm{~d}\left(\sigma_{f}=0.35\right)$ with the relevant displacement comparisons given in Figs.12b and c, or even farther right of $\mathrm{PF}_{2}$ bifurcation, as shown in Fig.11e $\left(\sigma_{f}=0.5\right)$. Moreover, with $\sigma_{f}=0$, both 1:1 and 2:1 resonant initiations in Fig.11f end up with a beating-type phenomenon and amplitude-modulated feature due to periodical energy transfer of 2:1 resonant interaction. This validates Fig.3 about how periodic 2:1 resonant amplitudes may originate from the nearby Hopf bifurcation at $\sigma_{f}=0.123$. Overall, such numerically observed prevailing role of 2:1 over 1:1 resonance is in qualitative agreement with that observed in nonlinear undamped free planar vibrations [11], where directly initiating the second symmetric mode at second crossover entails 2:1 resonant interaction involving first anti-symmetric mode, instead of the coexisting 1:1 resonant interaction involving second anti-symmetric mode. With regard to CPU time, all FD simulations in Fig.11 require a number of forcing periods $(>1500)$ to achieve steady responses longer than that $(<1000)$ for lower-sagged first-crossover cable (e.g., Fig.6).

Finally, it is certainly worth evaluating the actual internal detuning $\sigma$ parameter from a transient-free FD time series since the overall discussion of interrelated FD-MS solutions is based on $\sigma=0$. As an example, a Fourier-based analysis of the two simulations in Fig.11a is performed, and results of $1: 1$ and 2:1 resonant initiations are plotted in Figs.13a and b, respectively. Remarkably, both responses highlight two major peaks, with the amplitude $a_{r}$ being greater than $a_{s}$, which is in good accordance with the 2:1 resonant MS prediction in Fig.3 for $\sigma_{f}=$ -0.4. The corresponding two nonlinear frequency values are the same in both plots, i.e., 0.165 and $0.328 \mathrm{~Hz}$, thus providing a nearly perfect tuned 2:1 frequency ratio. Apart from confirming the actually predominant role of 2:1 resonance at second crossover, relying upon $\sigma=0$ in MS solutions appears reliable for MS-FD comparisons. 


\subsection{Discussion on Approximate/Exact and Planar/Non-Planar Cable Modeling}

For the sake of completeness and truth, straightforward FD analyses of more realistic cable models are now addressed shortly. Based on the same assigned control parameters and initial conditions, attention is paid to highlighting (i) the validity of approximate PDEs (1-2) vs. the exact ones [11] for planar motion, and (ii) the limitation of the approximate planar (2-D) modeling (herein considered) vs. the associated non-planar (3-D) one [7].

Regarding the first issue, the FD responses of approximate PDEs are compared with those of exact PDEs which are valid for also a larger amplitude range and a larger sagged cable. Comparisons for both uncoupled and coupled (constant- or varying-amplitude) solutions are made in terms of induced nonlinear dynamic tensions whose strains are spanwise non-uniform [9], thus being of remarkable engineering significance. By considering first-crossover cable, the benchmarking (mid-span) responses of dynamic tension $\tau$, normalized with respect to maximum static tension, are compared in Fig.14, whose left (right) column represents approximate (exact) PDEs' outcomes. Various cases of 1:1 resonant steady or modulated responses are considered, with Figs.14a, c, e (14b, d, f) corresponding to Figs.8b, 7f and 10b, respectively. The available maximum/minimum $\tau$ values of fixed-point MS solutions are also reported in Figs.14a and c with horizontal lines. Interestingly, Fig.14 highlights overall qualitative as well as quantitative agreements for both steadily constant-amplitude (a-d) and varying-amplitude (e-f) $\tau$ responses between MS-approximate, FD-approximate and FD-exact modeling, along with the compatibility of FD transient periods and features. Thus, for finite-amplitude vibrations of small-sagged cable, the MS/FD nonlinear tension responses are both valid and the associated approximate PDEs (12) can be used reliably.

Regarding the second issue, it is well known [1] that multiple internal resonances exist at crossovers involving also out-of-plane (denoted $w$ ) modes and planar vs. non-planar response scenarios $[3,4]$ differing from those predicted in Figs.2 and 3. Investigating them systematically in the analytical-numerical comparison perspective herein pursued would require extensive and 
heavy MS-FD analyses. We just limit ourselves to reconsidering both crossover cables to show how the modulated planar responses obtained with the 2-D model turn into steady non-planar responses when correctly considering the associated 3-D model [7] with the same planar/nonplanar damping $(\mu=.005)$. Focusing on $\sigma_{f}=0$, whose periodically amplitude-modulated FD $v$ responses based on planar model are given in Figs.10b and 11f, the corresponding FD $v(x=$ $0.50)$ and $w(x=0.26)$ responses based on non-planar model are plotted in Figs.15a, $\mathrm{c}$ and 15b, $\mathrm{d}$, for first- and second-crossover cables, respectively. It can be seen that in both responses, after some transients, the initiated planar amplitudes decrease up to low values, whereas substantial non-planar amplitudes are born from trivial values, exhibit some transients and then become ultimately steady. Correspondingly, the spatial displacement profiles visualized in Figs.16a and b show the characters of lowest symmetric (a) and anti-symmetric (b) out-of-plane mode, the coexisting non-planar $2: 1$ resonance actually predominating against the planar 1:1/2:1 resonances at first/second crossovers, respectively, and entailing overall regularization of cable response. Thus, the constrained planar model can be inadequate for such a systematic response analysis, and it is definitely advised to use the complete 3-D model for capturing, analytically and/or numerically, the actual non-planar resonant dynamics.

\section{SUMMARY AND CONCLUDING REMARKS}

Direct numerical FD-based simulations of PDEs governing geometrically nonlinear forced dynamics of suspended cables under primary external and 1:1 or 2:1 internal resonances have been carried out, in order to validate analytical MS-based predictions of associated finitedimensional ODEs. Different sagged horizontal cables at the first two crossovers involving mixed symmetric/anti-symmetric modal interactions have been analyzed, by relying on the approximate - kinematically non-condensed - planar modeling. With weakly quadratic/cubic

nonlinearities and multiple modal contributions, local scenarios of stable/unstable and uncoupled/coupled MS solutions have been evaluated by means of frequency response curves 
and bifurcations, accounting for also the influence of further control parameters. Towards the aim of interrelating analytical/numerical solutions, the corresponding amplitude-dependent damped forced resonant MS displacements have been constructed and further utilized as spatial initiations in FD simulations capturing fast/slow dynamics of a higher-dimensional model.

Depending on system control parameters and spatial initiations, space-time varying FD examinations reveal the actual existence of steady-state uncoupled (symmetric) vs. coupled (mixed) amplitude (displacement) MS solutions, along with meaningful transient evolution and dynamic-instability mode-transition characteristics. As regards computational CPU time efforts, directly initiating with proper MS-based spatial configurations makes the steady-state FD responses accessible with fewer transient periods than those necessary with conventional zero initiations. This allows undertaking large parametrical studies, which are needed for practical applications. The observed variability of spatial numerical outcomes - highlighting actual predominance of solely primary or primary/internally resonant dynamics - mainly depends on whether the initiated displacements/phases are zero, uncoupled or coupled, in contrast with the analytical solutions whose spatial distributions and relative phases are constrained to pertinent eigenfunctions and modulation equations, respectively.

With reference to a cable benchmarking point, comparisons of uncoupled/coupled (downward/upward) spatial MS-FD displacements highlight quantitative as well as qualitative agreement of multi-degree-of-freedom responses. Overall, direct numerical simulations thoroughly validate analytical predictions. Apart from the limitation concerned with considering the 2-D cable modeling, substantial parametric findings enable us to precisely identify the role of coexisting/competing (uncoupled vs. coupled) resonant dynamics, the actual jump phenomena with the relevant amplitude range, the predominant $2: 1$ vs. 1:1 (as well as non-planar vs. planar) internal resonance, the periodically amplitude-modulated coupled responses, the actual nonlinear frequencies, and the validity - through nonlinear dynamic tension comparisons - of kinematically approximate ODEs/PDEs, for small-sagged cables, against exact PDEs. 
It is worth emphasizing that, by accounting for multi-dimensional space-time dependent responses, direct numerical simulation and validation of analytical prediction are of the utmost importance for engineering design and practice. Besides systematically complementing the essential, analytically disclosed, system dynamics, numerical achievements furnish improved understanding of the basic mechanisms of dynamic responses, along with valuable information about possible prevalence of actually coexisting phenomena. It is felt that the present analyses may pave the correct methodological way for conducting a 3-D investigation of cable nonlinear forced vibrations involving a comprehensive description of out-of-plane/in-plane responses.

ACKNOWLEDGEMENT The first author wishes to acknowledge a Postdoctoral Fellowship from SAPIENZA University of Rome for this research.

\section{REFERENCES}

1. Rega G., Nonlinear dynamics of suspended cables. Part I: Modeling and analysis; Part II: Deterministic phenomena, ASME Applied Mechanics Reviews 57, 2004, 443-514

2. Pakdemirli M., Nayfeh S.A., Nayfeh A.H., Analysis of one-to-one autoparametric resonance in cables-discretization vs. direct treatment, Nonlinear Dynamics 8, 1995, 65-83

3. Rega G., Lacarbonara W., Nayfeh A.H., Chin C.M., Multiple resonances in suspended cables: Direct vs. reduced-order models, International Journal of Non-linear Mechanics 34, 1999, 901-924

4. Nayfeh A.H., Arafat H.N., Chin C.M., Lacarbonara W., Multimode interactions in suspended cables, Journal of Vibration and Control 8, 2002, 337-387

5. Arafat H.N., Nayfeh A.H., Non-linear responses of suspended cables to primary resonance excitations, Journal of Sound and Vibration 266, 2003, 325-354

6. Lacarbonara W., Rega G., Nayfeh A.H., Resonant nonlinear normal modes. Part I: Analytical treatment for structural one-dimensional systems, International Journal of Non-linear Mechanics $38,2003,851-872$

7. Srinil N., Rega G., Chucheepsakul S., Two-to-one resonant multi-modal dynamics of horizontal/inclined cables. Part I: Theoretical formulation and model validation, Nonlinear Dynamics 48, 2007, 231-252 
8. Srinil N., Rega G., Two-to-one resonant multi-modal dynamics of horizontal/inclined cables. Part II: Internal resonance activation, reduced-order models and nonlinear normal modes, Nonlinear Dynamics 48, 2007, 253-274

9. Srinil N., Rega G., The effects of kinematic condensation on internally resonant forced vibrations of shallow horizontal cables, International Journal of Non-linear Mechanics 42, 2007, 180-195.

10. Srinil N., Rega G., Chucheepsakul S., Large amplitude three-dimensional free vibrations of inclined sagged elastic cables, Nonlinear Dynamics 33, 2003, 129-154

11. Srinil N., Rega G., Chucheepsakul S., Three-dimensional nonlinear coupling and dynamic tension in the large amplitude free vibrations of arbitrarily sagged cables, Journal of Sound and Vibration 269, 2004, 823-852

12. Ni Y.Q., Lou W.J., Ko J.M., A hybrid pseudo-force/Laplace transform method for non-linear transient response of a suspended cable, Journal of Sound and Vibration 238, 2000, 189-214

13. Tjavaras A.A., Zhu Q., Liu Y., Triantafyllou M.S., Yue D.K.P., The mechanics of highlyextensible cables, Journal of Sound and Vibration 213, 1998, 709-737

14. Koh C.G, Rong Y, Dynamic analysis of large displacement cable motion with experimental verification, Journal of Sound and Vibration 272, 2004, 187-206

15. Zhou Q., Nielsen S.R.K., Qu W.L., Semi-active control of three-dimensional vibrations of an inclined sag cable with magnetorheological dampers, Journal of Sound and Vibration 296, 2006, 1-22

16. Abhyankar N.S., Hall E.K., Hanagud S.V., Chaotic vibrations of beams: numerical solution of partial differential equations, ASME Journal of Applied Mechanics 60, 1993, 167-173

17. Essebier S., Baker G., Computational techniques for nonlinear dynamics of continuous systems, ASCE Journal of Engineering Mechanics 121, 1995, 1193-1999

18. Abe A., On non-linear vibration analyses of continuous systems with quadratic and cubic non-linearities, International Journal of Non-linear Mechanics 41, 2006, 873-879

19. Gattulli V., Martinelli L., Perotti F., Vestroni F., Nonlinear oscillations of cables under harmonic loading using analytical and finite element models, Computer Methods in Applied Mechanics and Engineering 193, 2004, 69-85

20. Irvine H.M., Cable Structures, 1981, MIT Press, Cambridge, MA

21. Rega G., Srinil N., Non-linear hybrid-mode resonant forced oscillations of sagged inclined cables at avoidances, ASME Journal of Computational and Nonlinear Dynamics, 2007, in press.

22. Seydel, R., Practical Bifurcation and Stability Analysis, 1994, Springer, New York 


\section{Figure}

\section{List of Figures}

1 Schematic suspended cable model (a) and orthonormalized $v$ eigenfunctions of resonant symmetric/anti-symmetric $(s / r)$ modes at first (b) and second (c) crossovers.

2 MS-based frequency-response curves and bifurcations of 1:1 resonant first-crossover cable with the effect of varying parameters: (a) coupled $a_{r}$, (b) coupled $a_{s}$, (c) uncoupled $a_{s}$.

3 MS-based frequency-response curves and bifurcations of 1:1 vs. 2:1 resonant second-crossover cable: (a) coupled $a_{r}$, (b) coupled $a_{s}$.

4 MS-based spatial $\left(\mathrm{Y}^{+}, \mathrm{Y}^{-}\right)$nonlinear $v$ dynamic configurations: (a) uncoupled and (b) coupled solutions of 1:1 resonant first-crossover cable, coupled solutions of (c) 1:1 and (d) 2:1 resonant second-crossover cable.

$5 \quad$ FD-based nonlinear time $v$ transient/steady responses at $x=0.5$ of 1:1 resonant first-crossover cable: (a-b) $\mu=.05, F=.005, \sigma_{f}=0.1$ with (a) zero or (b) uncoupled MS spatial $\left(\mathrm{Y}^{+}, \mathrm{Y}^{-}\right)$initiations; (c-d) $\mu=$ $.005, F=.005$, (c) $\sigma_{f}=0.1$ or (d) $\sigma_{f}=-0.3$ with zero initiations; (e-f) $\mu=.005, F=.01, \sigma_{f}=0.1$ with (e) zero or (f) uncoupled MS spatial $\left(\mathrm{Y}^{-}\right)$initiations.

6 FD-based nonlinear time $v$ transient/steady responses at $x=0.2$ (black) and 0.8 (grey), and corresponding steady $v$ displacement profiles of 1:1 resonant first-crossover cable with $\mu=.005, F$ $=.005, \sigma_{f}=-0.1$ : (a) zero, $(\mathrm{b}, \mathrm{c})$ uncoupled MS spatial $\left(\mathrm{Y}^{-}, \mathrm{Y}^{+}\right)$and $(\mathrm{d}, \mathrm{e})$ coupled MS spatial $\left(\mathrm{Y}^{-}, \mathrm{Y}^{+}\right)$ initiations.

7 A comparison of steady $\left(\mathrm{Y}^{+}, \mathrm{Y}^{-}\right)$nonlinear coupled $v$ displacement profiles of 1:1 resonant firstcrossover cable with influence of varying control parameters: solid lines (circles) denote MS (FD) solution.

$8 \quad$ FD-based nonlinear time $v$ transient/steady responses at $x=0.2$ (black) and 0.8 (grey), and a comparison of steady $\left(\mathrm{Y}^{+}, \mathrm{Y}^{-}\right)$nonlinear uncoupled $v$ displacement profiles of 1:1 resonant firstcrossover cable with $\mu=.005$ : (a) $F=.005, \sigma_{f}=0$, (b) $F=.01, \sigma_{f}=0.1$; solid lines (circles) denote MS (FD) solution.

$9 \quad$ FD-based nonlinear time $v$ transient/steady responses at $x=0.2$ (black) and 0.8 (grey) of 1:1 resonant first-crossover cable below actual jump phenomenon to uncoupled response: (a) $\mu=.05, F=.005$ and (b) $\mu=.005, F=.005$.

10 FD-based nonlinear time $u$ (a) and $v$ (b) responses at $x=0.2$ (black) and 0.8 (grey) of 1:1 resonant first-crossover cable showing periodically amplitude-modulated modal interactions with $\sigma_{f}=0, \mu=$ $.005, F=.01$.

11 FD-based nonlinear time $v$ steady responses at $x=0.26$ of second-crossover cable with $\mu=.005, F$ $=.005$ : (a) $\sigma_{f}=-0.4$, (b) $\sigma_{f}=-0.2$, (c) $\sigma_{f}=0.2$, (d) $\sigma_{f}=0.35$, (e) $\sigma_{f}=0.5$, (f) $\sigma_{f}=0$; solid lines (circles) denote 1:1 (2:1) resonant MS-based spatial initiations.

12 A comparison of steady $\left(\mathrm{Y}^{+}, \mathrm{Y}^{-}\right)$nonlinear coupled $v$ displacement profiles of predominating 2:1 resonant second-crossover cable in Fig.11: (a) $\sigma_{f}=-0.2$, (b) $\sigma_{f}=0.2$, (c) $\sigma_{f}=0.35$; solid lines (circles) denote FD solution with 1:1 (2:1) resonant MS-based spatial initiations; dashed lines denote MS solution.

13 Fourier-based frequency analysis of steady responses in Fig.11a: (a) 1:1 and (b) 2:1 resonant MSbased spatial initiations.

14 Direct FD simulations of cable nonlinear dynamic tensions at mid-span based on approximate (a, c, e) vs. exact (b, d, f) planar modeling: (a, b), (c, d) and (e, f) correspond to Figs.8b, $7 \mathrm{f}$ and 10b, respectively; horizontal lines in (a, c) denote corresponding maximum/minimum MS results.

15 Direct FD simulations of cable $v$ and $w$ responses at mid-span $(\mathrm{a}, \mathrm{b})$ and quarter-span $(\mathrm{c}, \mathrm{d})$ based on approximate 3-D modeling: (a, b) and (c, d) correspond to first (Fig.10b) and second (Fig.11f) crossover cables, respectively. 


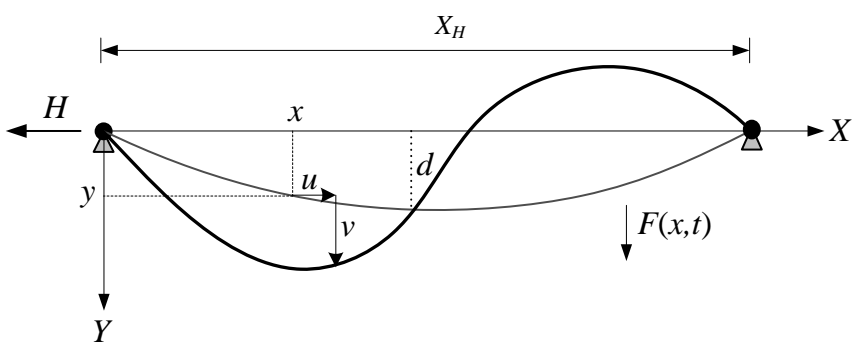

(a)
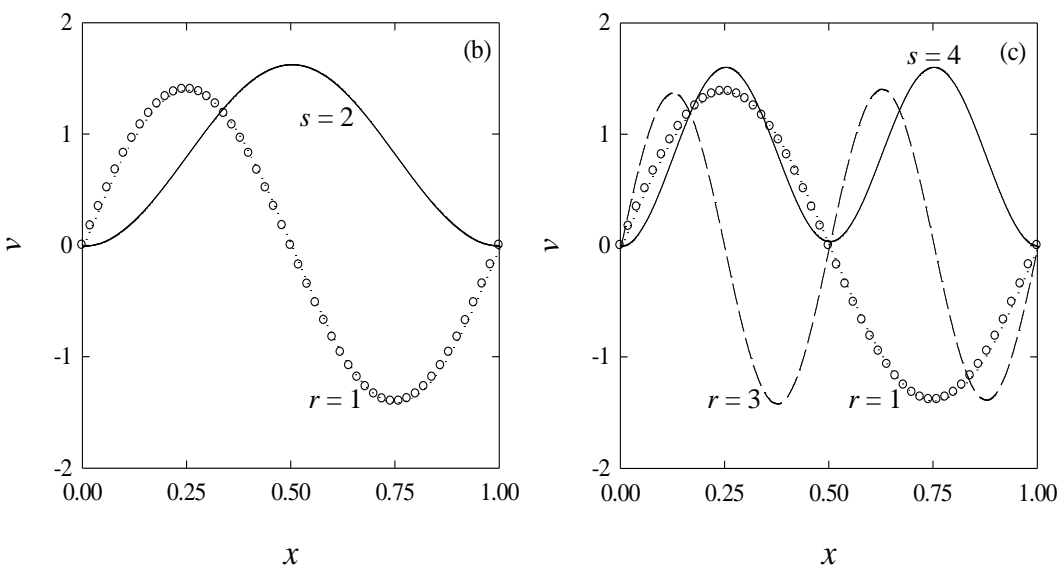

Figure 1 

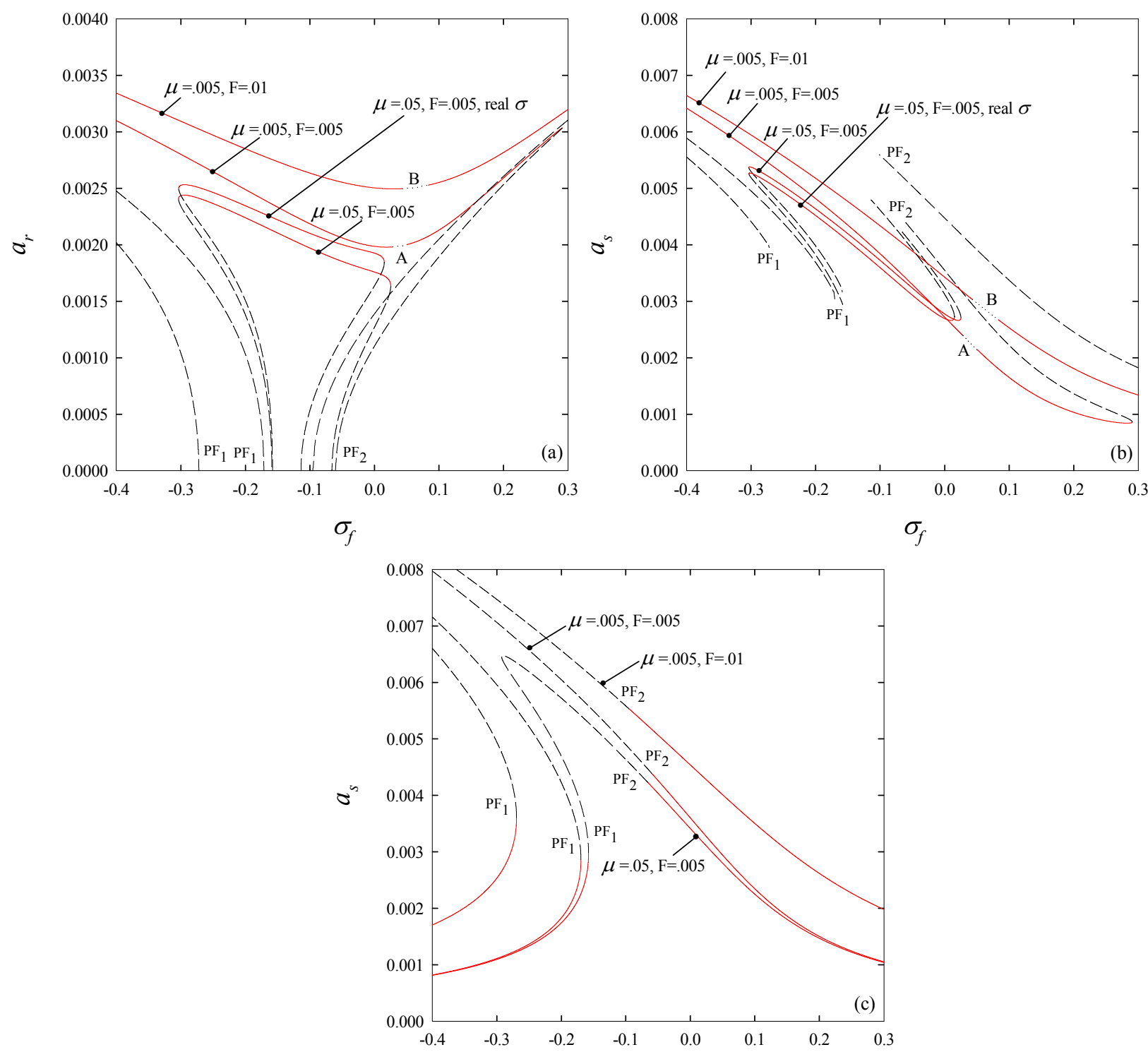

$\sigma_{f}$

Figure 2 

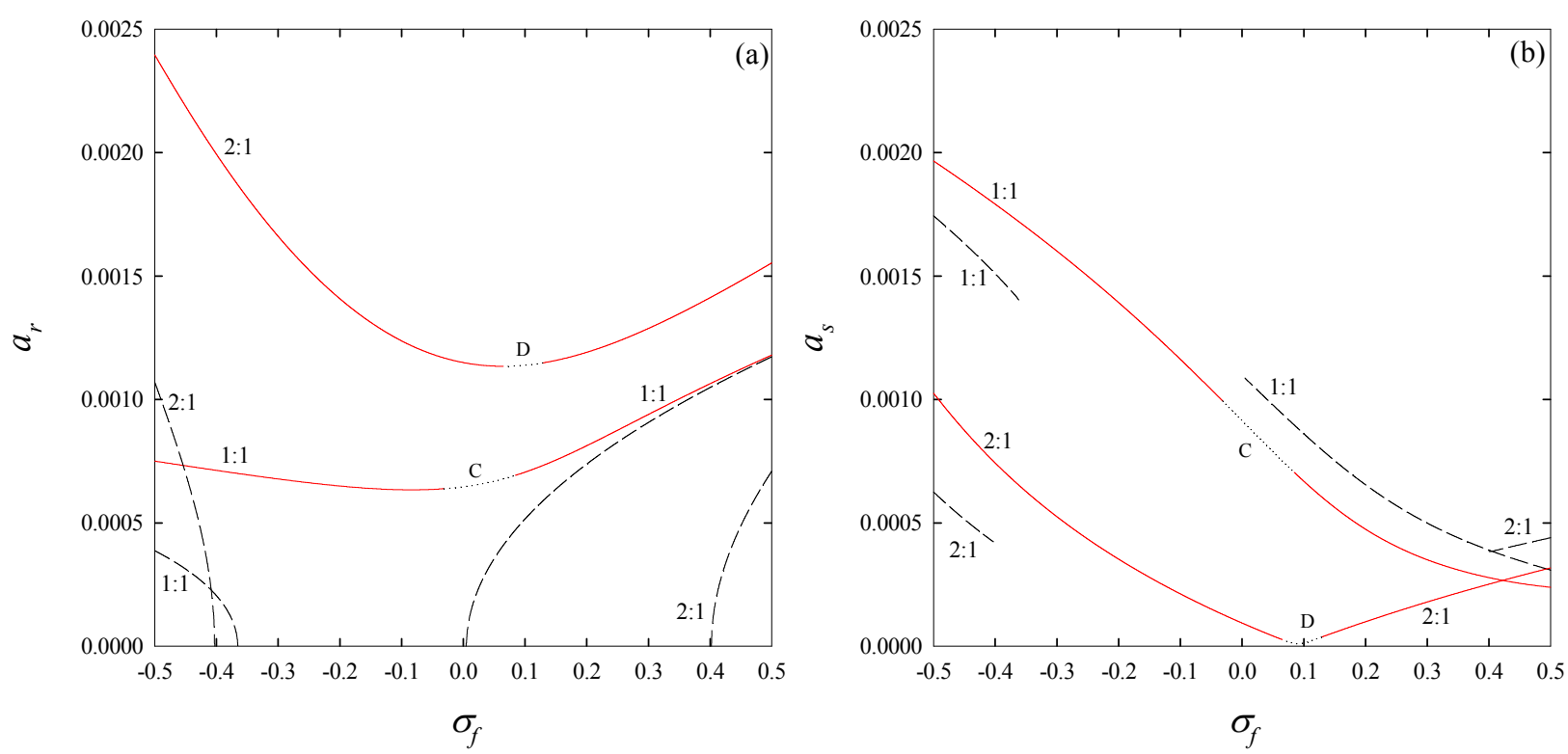

$\underline{\text { Figure } 3}$
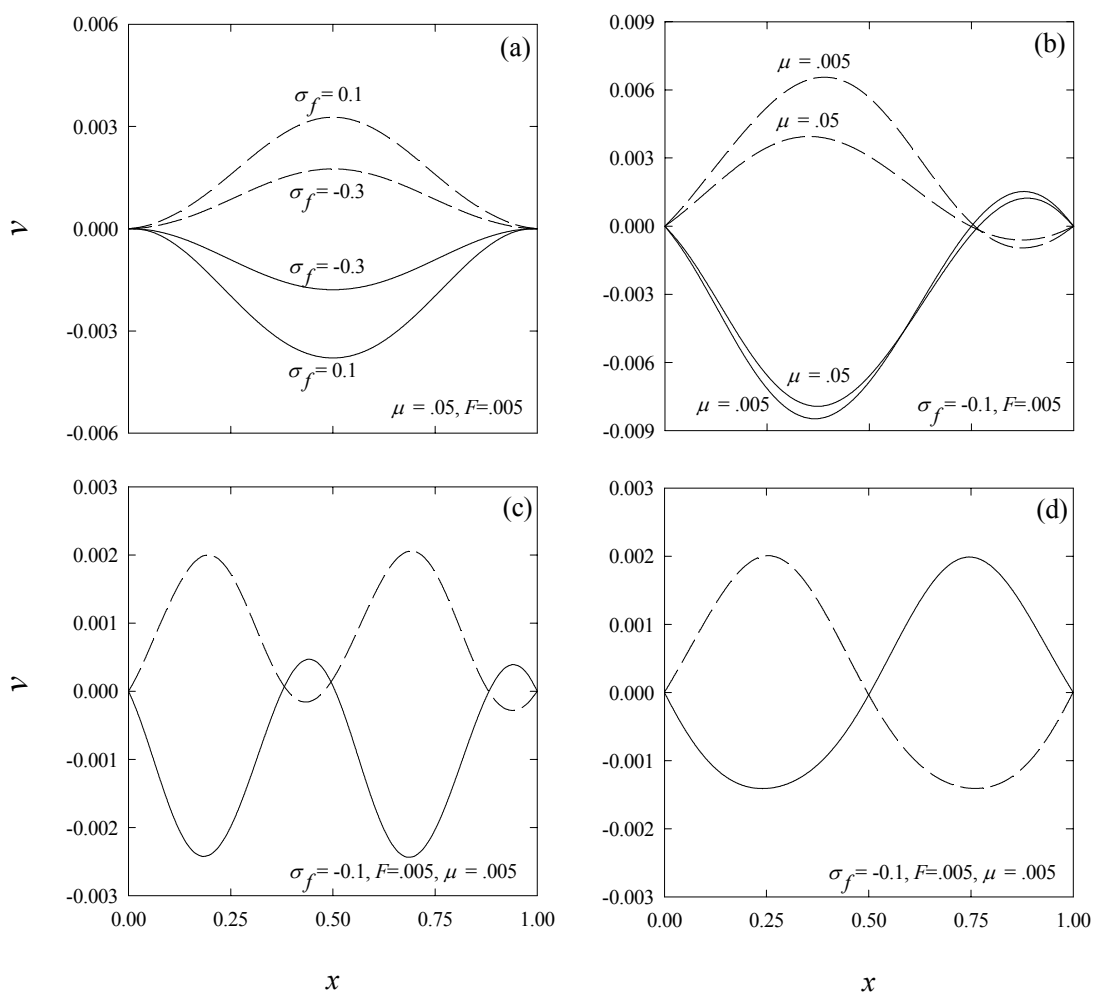

Figure 4 

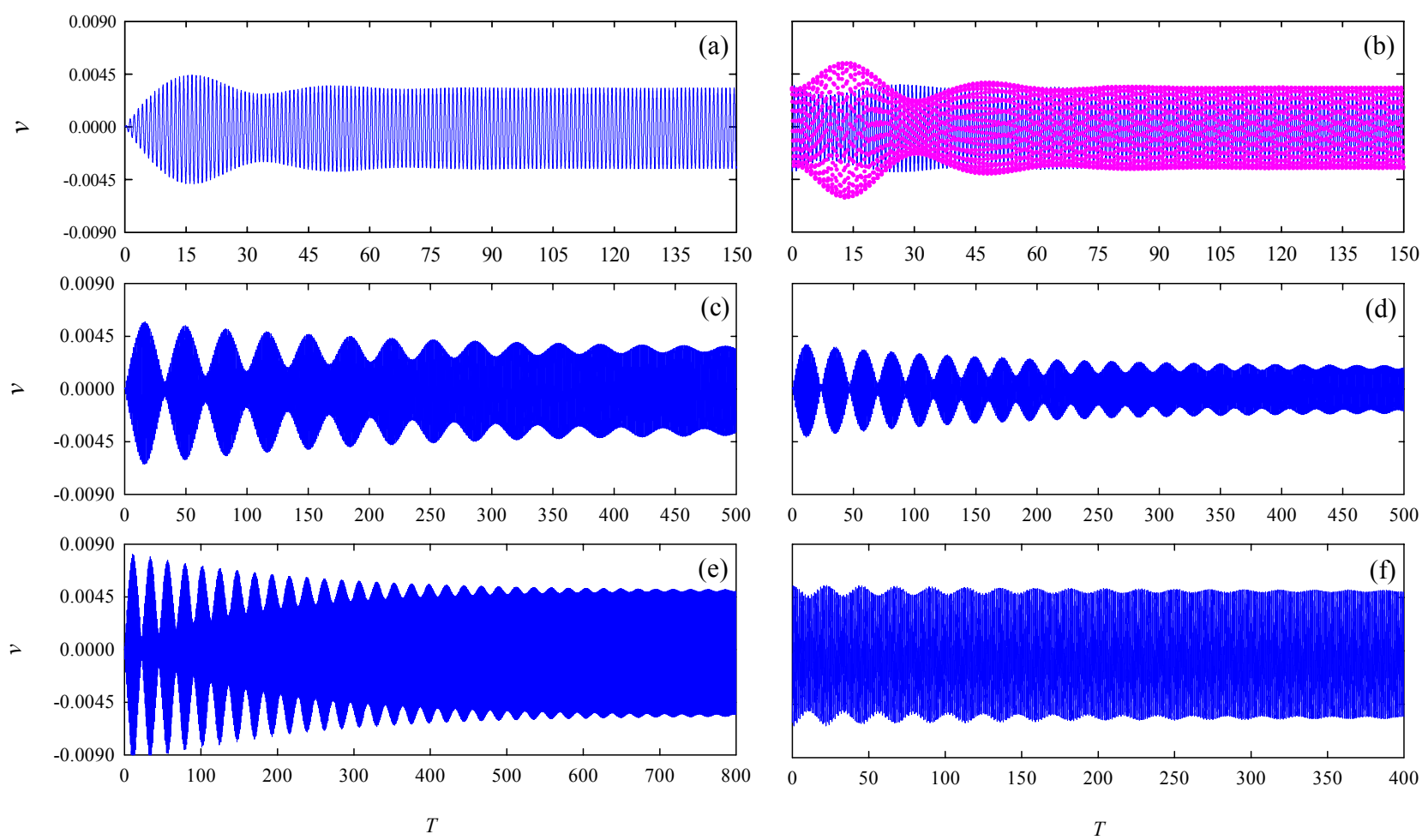

\section{Figure 5}



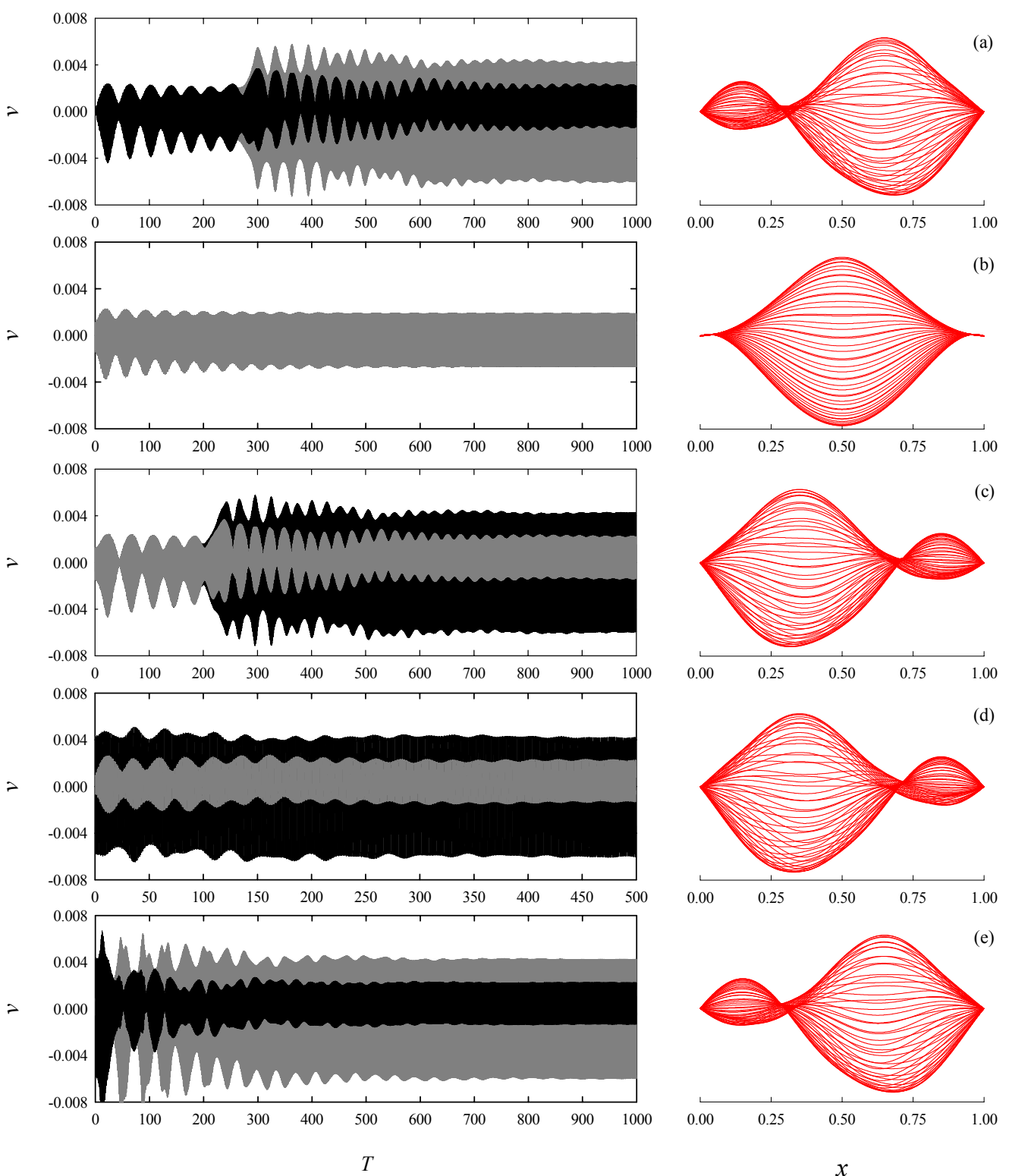

Figure 6 

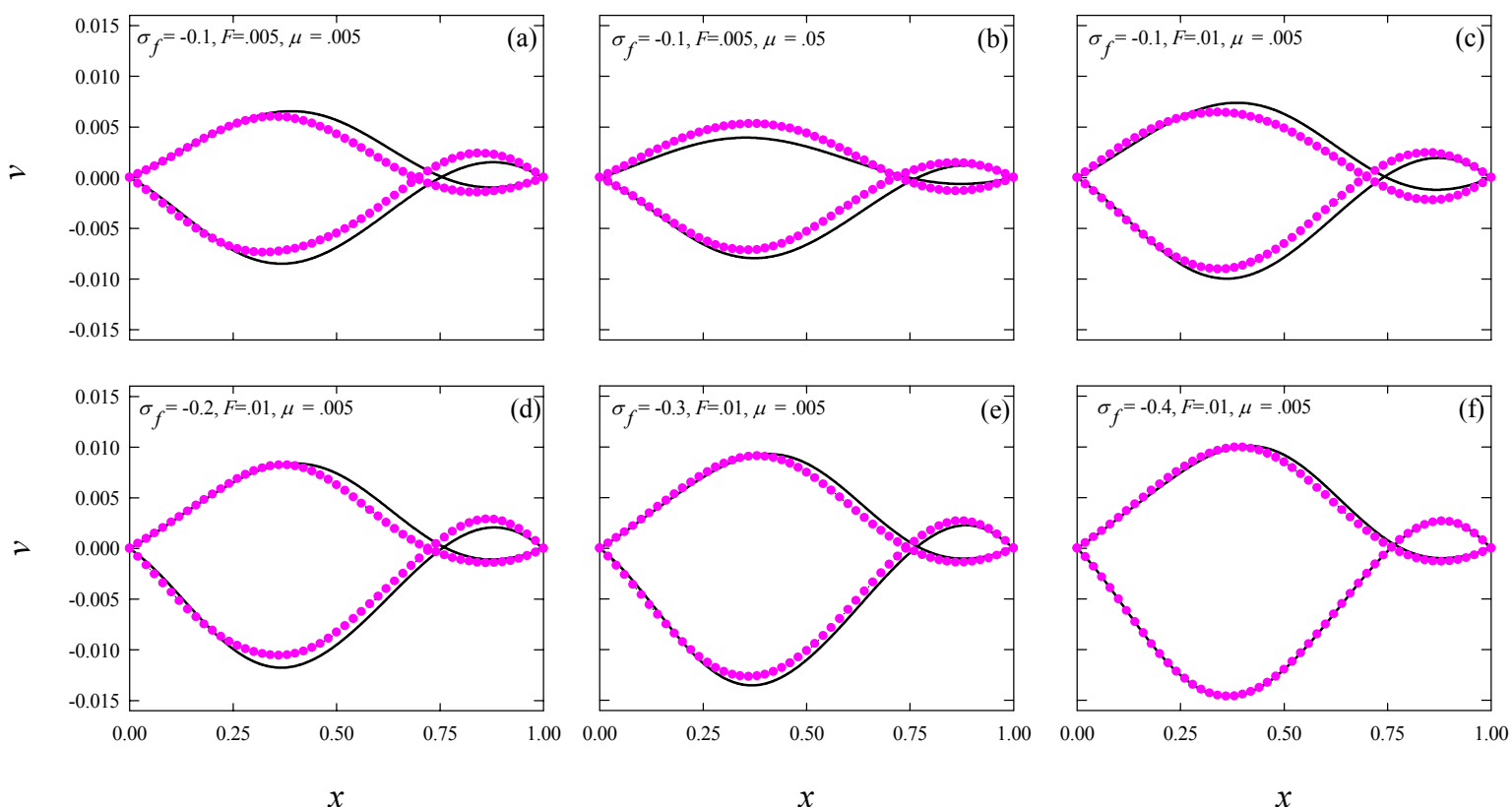

Figure 7 

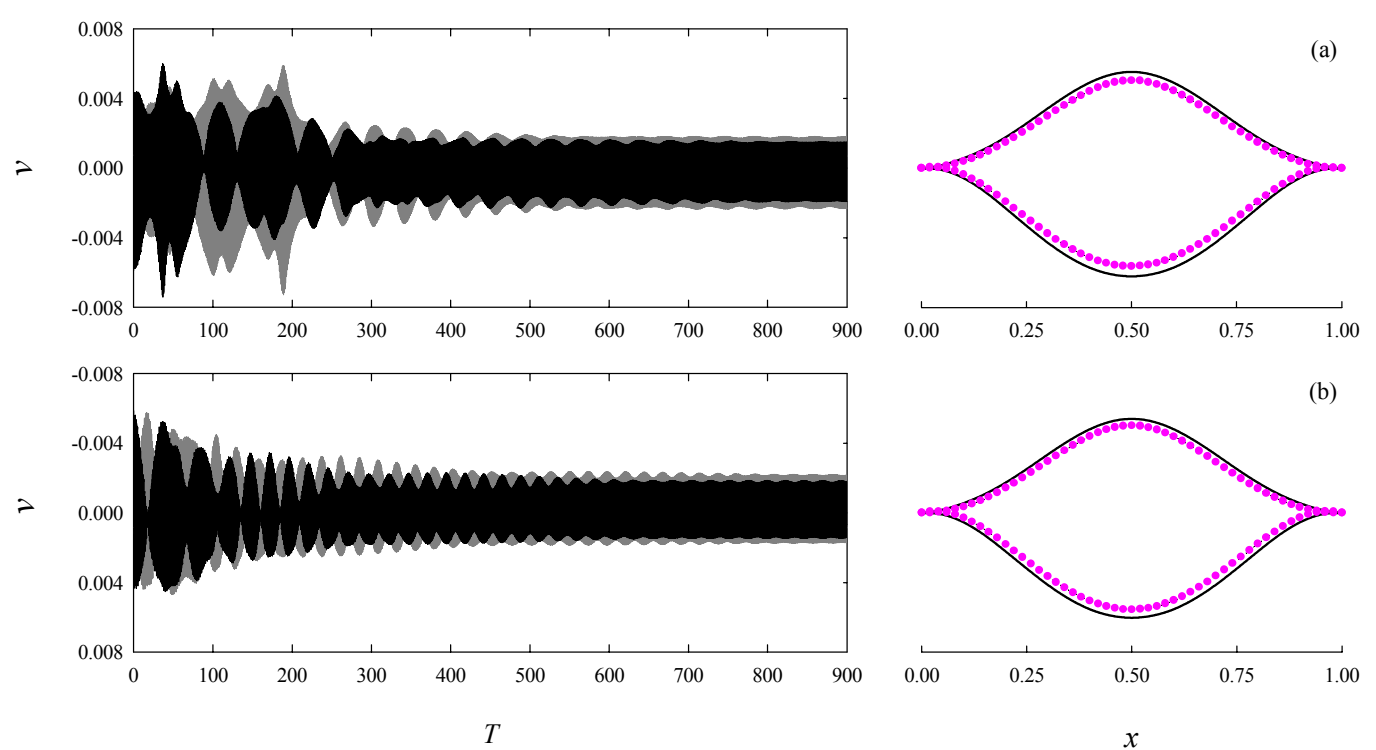

Figure 8
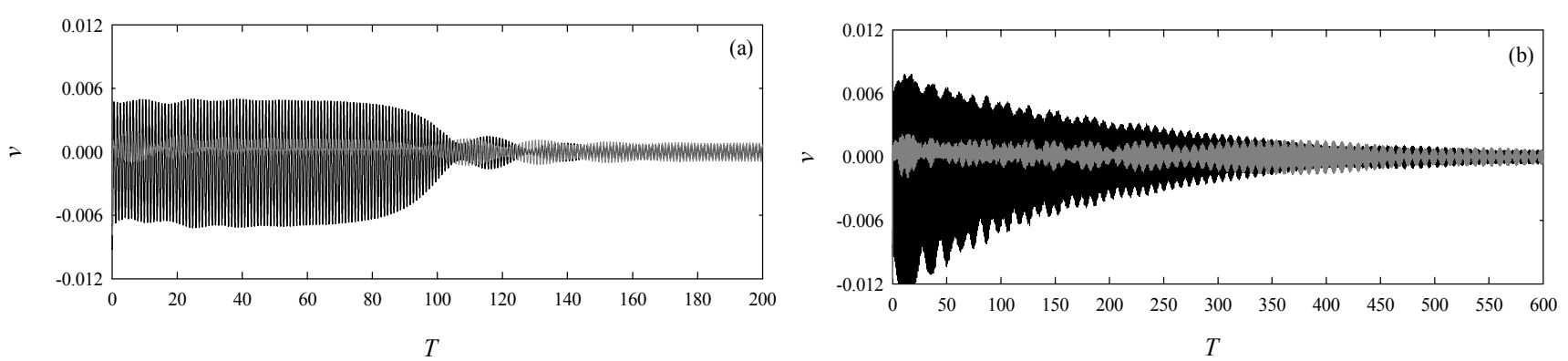

Figure 9
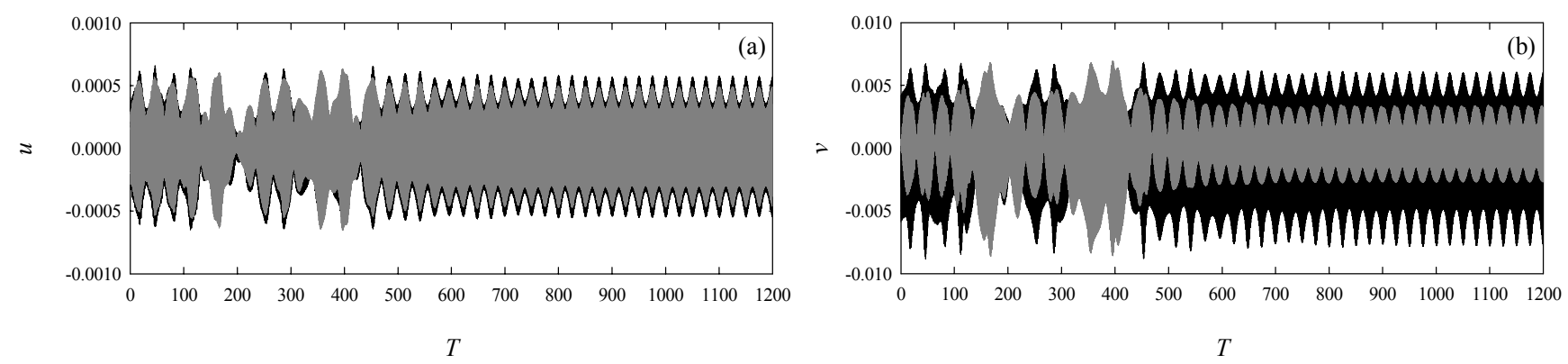

\section{Figure 10}



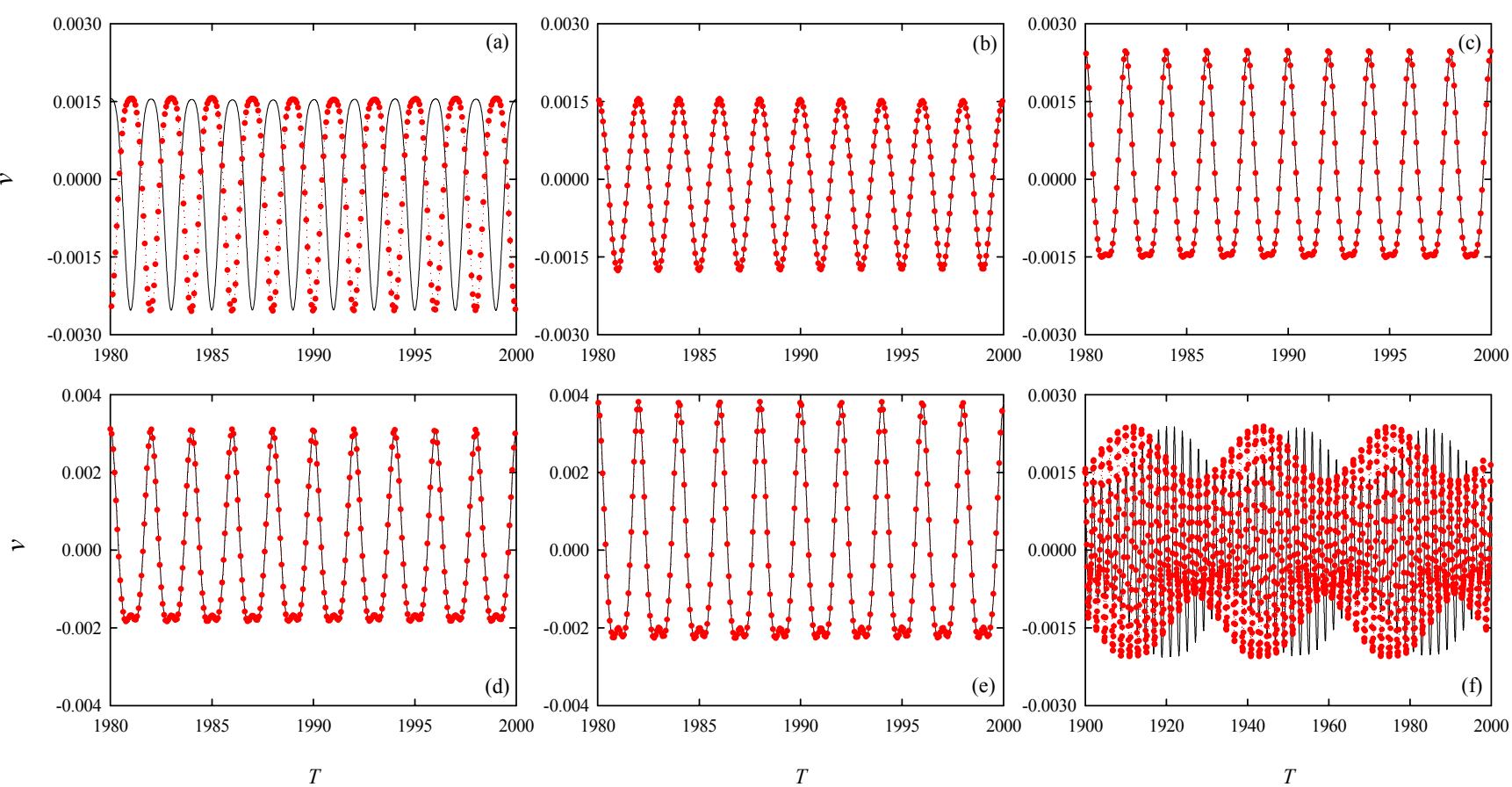

Figure 11
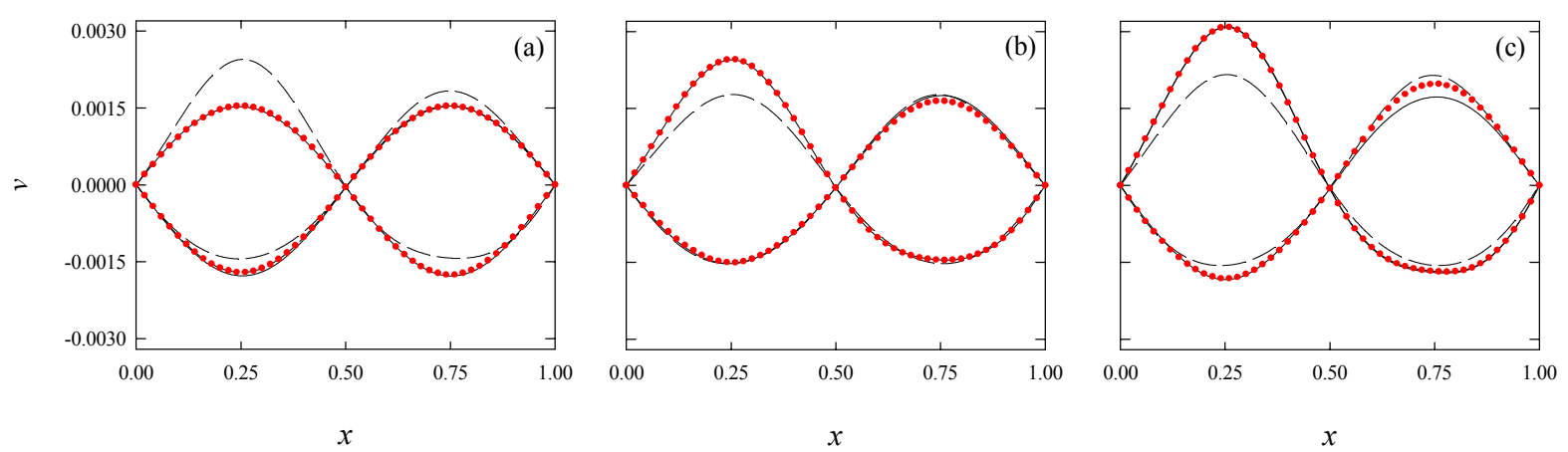

Figure 12 

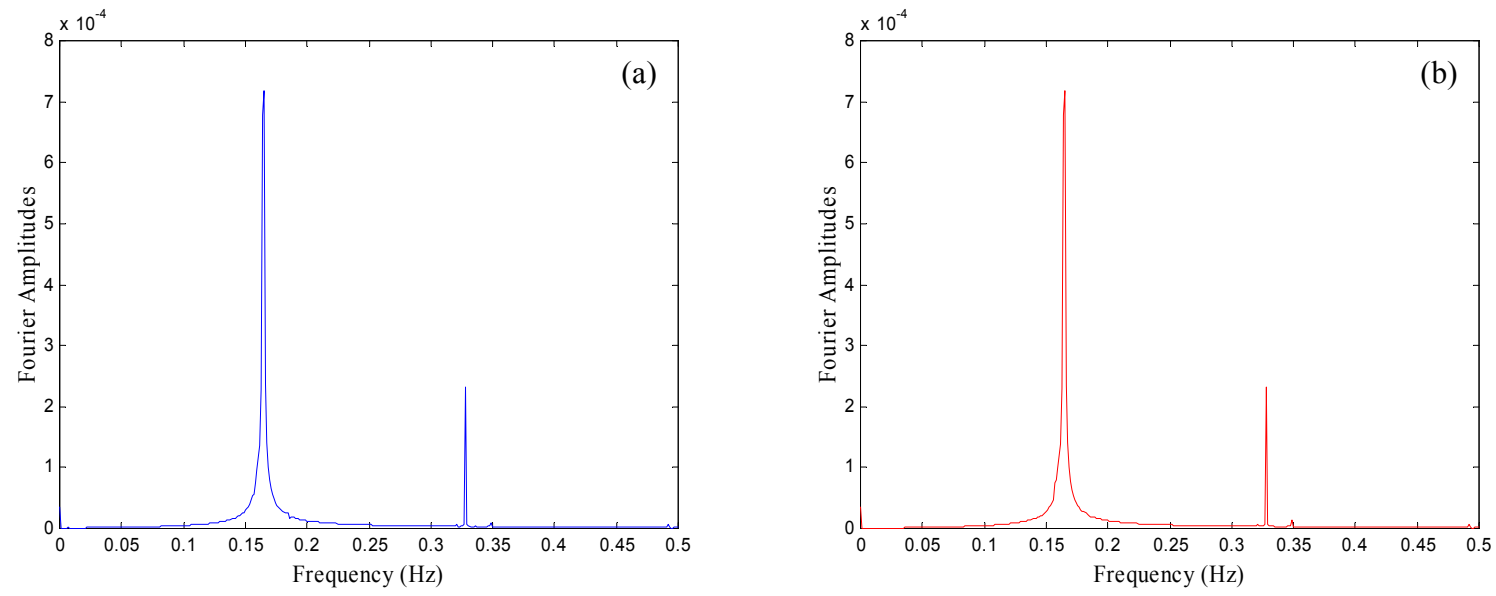

Figure 13 
Approximate Model

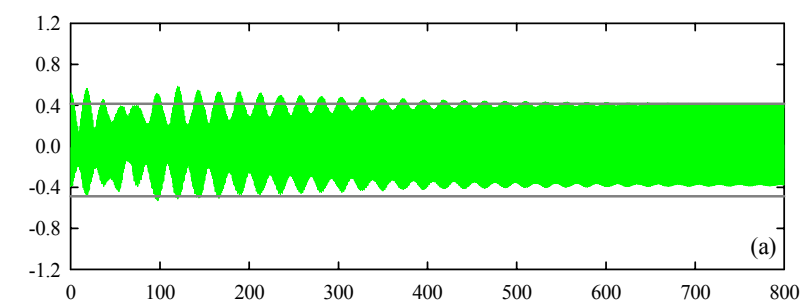

$\omega$
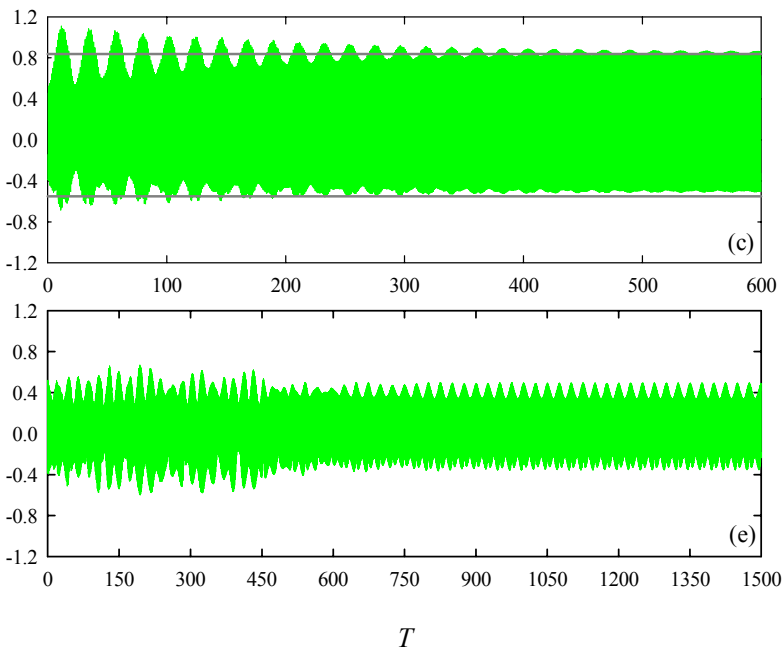

Exact Model
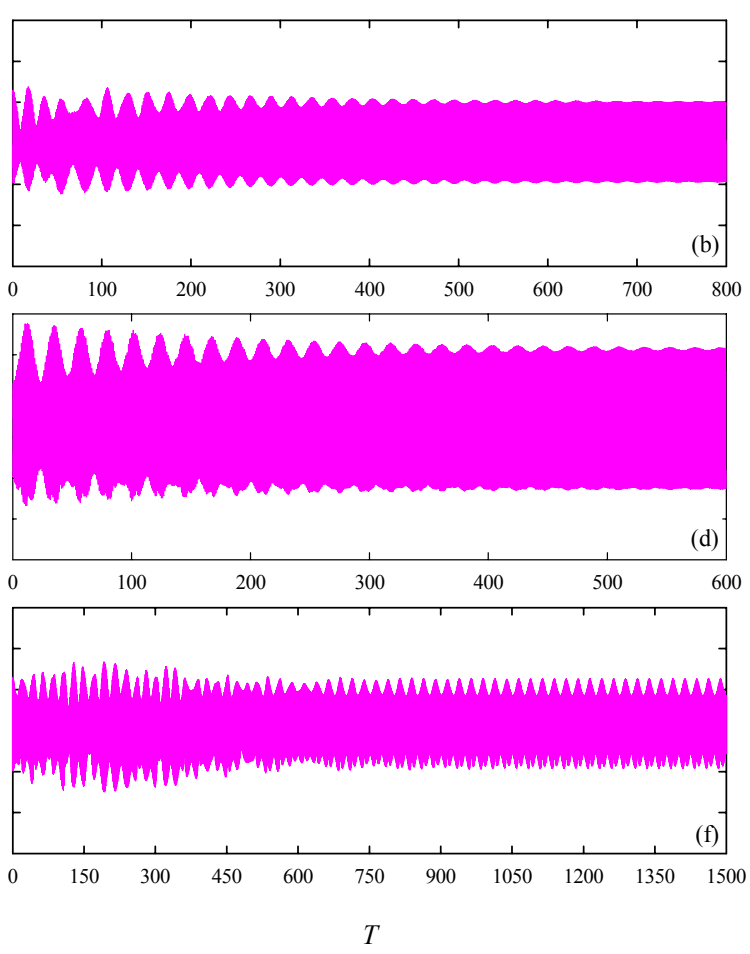

$\underline{\text { Figure } 14}$ 

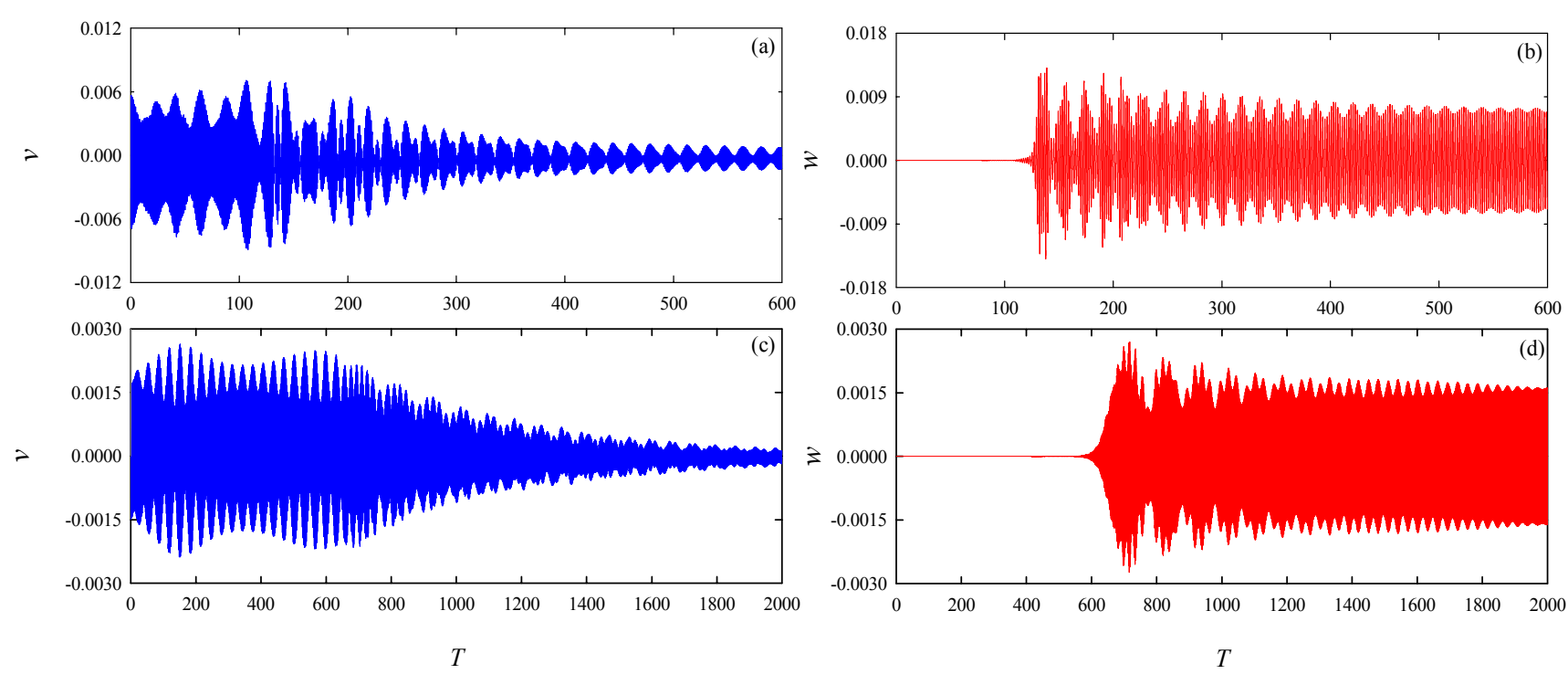

Figure 15
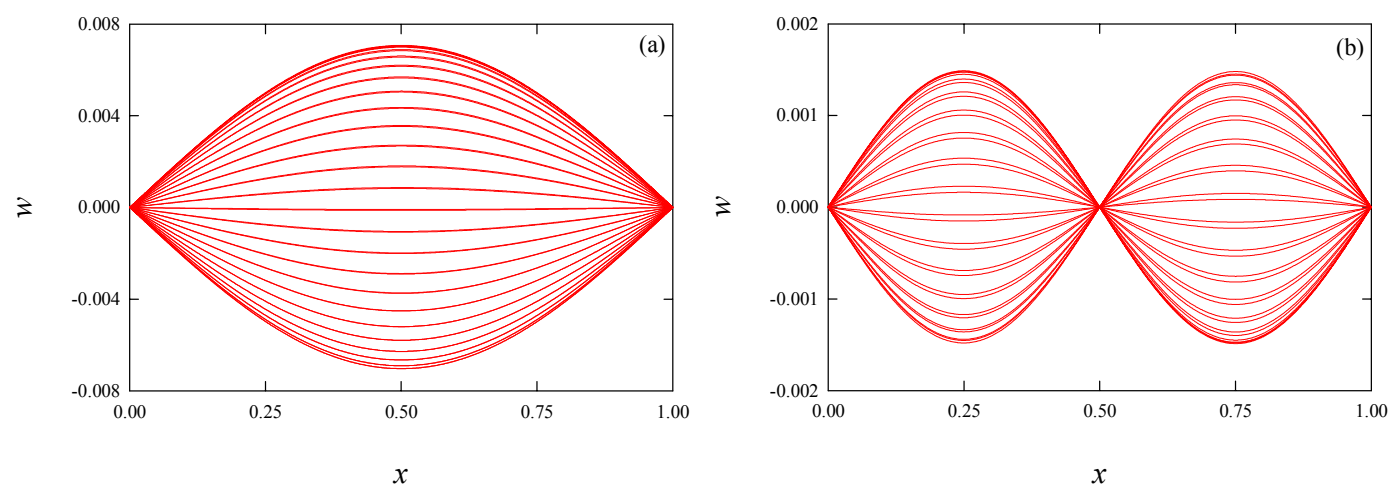

$\underline{\text { Figure } 16}$ 